\title{
Symmetric Hyperbolic Linear Differential Equations
}

\author{
By K. O. FRIEDRICHS
}

The present paper is concerned with symmetric systems of linear hyperbolic differential equations of the second order. The existence of a solution of Cauchy's initial problem will be proved under weak conditions. It will further be proved that the solution is differentiable in a sense to be specified if the right member and the coefficients of the equation possess appropriate differentiability properties.

This treatment uses the "Energy Integral" inequalities as the primary tool, together with simple facts from the theory of Hilbert space.

The energy integral was used by $\mathrm{H}$. Weber [1] to prove the uniqueness of the solutions of Maxwell's equations in the whole space and by Hadamard in 1900 [2] to prove the uniqueness of the solution of a mixed problem for the wave equation.

We shall briefly describe the latter problem. Let $\S_{0}$ denote a region in the $x, y$-plane and let $\delta_{t}$ consist of the points $(x, y, t)$ in space for which the projection $(x, y, 0)$ lies in $s_{0}$. Suppose a function $u(x, y, t)$ defined in the half-cylinder swept out by all segments $\varsigma_{t}$ for $t \geq 0$ satisfies the differential equation

$$
u_{i t}-u_{x x}-u_{y y}=0
$$

vanishes at the boundary of $s_{t}-$ or has vanishing normal derivatives thereand vanishes together with the derivative $u_{t}$ on the initial surface $\delta_{0}$. Then the relation

$$
\frac{d}{d t} \iint_{s_{t}}\left[u_{t}^{2}+u_{x}^{2}+u_{y}^{2}\right] d x d y=2 \iint_{s_{t}} u_{t}\left[u_{t t}-u_{x x}-u_{y y}\right] d x d y=0
$$

shows that the energy integral

$$
\frac{1}{2} \iint_{s_{t}}\left[u_{t}^{2}+u_{x}^{2}+u_{y}^{2}\right] d x d y
$$

is constant. Since this expression vanishes initially, it vanishes for all $t>0$. Hence $u \equiv 0$.for $t>0$ follows.

Zaremba [3] observed in 1915 that a similar argument could be used to prove the uniqueness of the solution of the wave equation in domains partially bounded by characteristic surfaces. The same fact was observed by Rubinowicz [4] in 1920 and Lewy [5A] in 1928. These two authors also deduced that the solution of the wave equation depends upon the initial data on only a section of the 
initial surface. Furthermore, they concluded from this fact that in processes governed by the wave equation disturbances are propagated with finite speed.

Specifically, let us consider the characteristic domain $R_{0}$ associated with a point $\left(x_{0}, y_{0}, t_{0}\right)$ with $t_{0}>0$,

$$
0 \leq t \leq t_{0}-\left[\left(x-x_{0}\right)^{2}+\left(y-y_{0}\right)^{2}\right]^{1 / 2} .
$$

Denoting by $\delta_{t}$ the cross-section of this domain with the plane $t=$ constant, and by $B_{t}$ the boundary of $\delta_{t}$, one derives the relation

$$
\begin{aligned}
\iint_{s_{t}}\left[u_{t}^{2}+u_{x}^{2}+u_{y}^{2}\right] d x d y+\int_{0}^{t_{2}} \int_{\mathbb{B} t}\left[\left(u_{x}-x_{n} u_{t}\right)^{2}\right. & \left.+\left(u_{y}-y_{n} u_{t}\right)^{2}\right] d s d t \\
& =\iint_{s_{0}}\left[u_{t}^{2}+u_{x}^{2}+u_{y}^{2}\right] d x d y,
\end{aligned}
$$

in which $d s$ is the arc differential and $\left(x_{n}, y_{n}\right)$ the exterior normal at any point of $B_{t}$. If $u=u_{t}=0$ on $S_{0}$, it follows that $u=0$ in the whole characteristic region $\mathbb{R}_{0}$. In particular, it is seen that the value of a solution at the point $\left(x_{0}, y_{0}, t_{0}\right)$ depends only on the data in the "domain of dependence" $S_{0}$.

Arguments of this type were also used to prove the uniqueness of solutions of linear hyperbolic equations with non-constant coefficients [5A].

The question of continuable initial conditions can also be answered with the aid of energy integrals. The type of conditions imposed on the data in the initial domain, e.g. $\delta_{0}$, are said to be "continuable" if a solution of the differential equation exists which satisfies conditions of the same type in appropriate spacelike domains, e.g. $S_{t}, t>0$. A theorem about the existence of the solution cannot be considered a satisfactory expression of Huygens' principle in the wider sense, as formulated by Hadamard, unless the conditions imposed on the initial data are continuable.

From energy integral considerations one infers that quadratic integrability of the function $u$ and its derivatives up to any order $r$ is a continuable condition, provided one admits solutions in a sufficiently wide sense. Continuity and existence of continuous derivatives of any order is implied by these conditions if the order $r$ of quadratically integrable derivatives is high enough. This was pointed out - in connection with the equation $u_{t \ell}-u_{x x}-u_{y \nu}=0$ as typical -by H. Lewy and the author in $1932[5 \mathrm{~B}]$.

Inequalities derived from energy integral identities can be used to establish the existence of the solutions of linear, and even nonlinear, hyperbolic partial differential equations. ${ }^{1}$ Such inequalities were used by Schauder [6], who in

${ }^{1}$ This possibility was indicated in the above-mentoned note $[5 \mathrm{~B}]$, where the author announced a proof of the existence of the solution of the initial problem for nonlinear hyperbolic equations of second order involving continuable initial data. A complete manuscript concerning functions of three variables existed early in 1932. The method of energy integrals was combined with the method of finite differences. The manuscript was not published because the treatment, in particular of the initial data, was very involved. 
1935 gave a proof of the existence of the solution of a nonlinear hyperbolic equation of second order and any number of variables. In particular, these inequalities involve the square integrals of the derivatives of the functions $u$ as well as of the coefficients of a sufficiently high order. Schauder approximates the coefficients and the initial data by analytic functions and uses the solution of CauchyKowalewski. An important step in this approach consists in showing that this solution of the approximate problem exists in a fixed domain, independent of the approximation.

Schauder does not establish the fact that the initial data are continuable. A remedy for this defect was described by Frankl [8] in 1937, who established the proper energy integral inequalities under sufficiently economical differentiability conditions. In his approximation procedure, which differs somewhat from that of Schauder, he employes methods of Sobolev and Petrovskii. Under continuable conditions which are definitely less restrictive than those needed by Frankl, the unique existence of solutions of nonlinear hyperbolic equations of second order was established by Sobolev [11] in $1939 .^{2}$

A very peculiar approach to Cauchy's problem for hyperbolic equations was developed by Petrovskii [9] in 1937. He considers general hyperbolic systems and employs square integrals after Fourier transformation. While energy integral identities are essentially associated with symmetric hyperbolic systems, no such restriction is necessary in Petrovski's approach. Severe limitations of this approach, concerning topological properties of the $n$-dimentional sphere, were pointed out by Leray.

A new approach to hyperbolic equations was recently developed by Leray [13], who, in a certain sense, reduces general to symmetric hyperbolic systems and then employs energy integrals.

In the present paper we shall treat only linear symmetric hyperbolic systems. We shall show that the existence of the solution-in a certain generalized senseof Cauchy's problem can be deduced from the simplest facts in the theory of the Hilbert space. Furthermore we shall prove a differentiability theorem with the aid of the solution of a simple finite difference equation. Continuous differentiability of the solution in the ordinary sense can then immediately be deduced if the data and the coefficients possess appropriate differentiability properties. There is little doubt that Schauder's arguments could also be employed in

2There are various approaches to the theory of hyperbolic differential equations in which the energy integral method is not used. This applies in particular to the theory of equations for functions of two variables. (See the fortheoming report by P. Lax [191.) Various approaches to the theory of linear hyperbolic partial differential equations related to that developed by Hadamard [15] and Riesz [16] have been treated by various authors. We mention that a particular such approach developed in 1936 by Sobolev [7B] was employed by Christianovitch [10] in 1937 for the treatment of nonlinear hyperbolic equations. A related approach was used by Fourès-Bruhat [18] in 1952 for a more extended treatment of such nonlinear equations.

Extensive work on linear hyperbolic equations, in particular of higher than second order, was done by Bureau. For a report of his work and work of others on hyperbolic equations see [17]. 
obtaining the main results of this paper. Nevertheless, it was felt to be of sufficient interest to show that these results could be obtained through direct constructions, without using approximation by analytic functions and the theorem of Cauchy and Kowalewski.

We proceed to give an outline of our methods and results.

As mentioned above we are not concerned with one equation of second order, but with a symmetric system of first order. We denote by $x=\left\{x^{1}, \cdots, x^{m}\right\}$ the points in the $m$-dimensional space and introduce the differentiation $D_{\mu}=$ $\partial / \partial x^{\mu}, \mu=1, \cdots, m$. With the aid of $m+1$ matrices

$$
\begin{aligned}
A^{\mu} & =\left\{A_{11}^{\mu}, A_{12}^{\mu}, \cdots, A_{p p}^{\mu}\right\}, \quad \mu=1, \cdots, m, \\
B & =\left\{B_{11}, B_{12}, \cdots, B_{p p}\right\},
\end{aligned}
$$

which are given functions of $x$, we introduce the differential operator

$$
E=A^{1} D_{1}+\cdots+A^{m} D_{m}+B .
$$

It transforms systems $u=u(x)$ of $p$ functions,

into systems

$$
u=\left\{u_{1}, \cdots, u_{p}\right\}
$$

here

$$
E u=A^{1} D_{1} u+\cdots+A^{m} D_{m} u+B u
$$

$$
D_{\mu} u=\left\{D_{\mu} u_{1}, \cdots, D_{\mu} u_{p}\right\}
$$

Giving a system $f=\left\{f_{1}, \cdots, f_{m}\right\}=f(x)$ as the "right member," we formulate the differential equation as

$$
E u=f .
$$

The operator $E$ is called hyperbolic at a point $y$, in the sense of Petrovskii [9], if among the systems of $m$ numbers,

$$
\xi=\left\{\xi_{1}, \cdots, \xi_{m}\right\}
$$

there is a particular one, $\xi^{0}$, such that the set of matrices

$$
\sum_{\mu} \xi_{\mu} A^{\mu}-\lambda \sum_{\mu} \xi_{\mu}^{0} A^{\mu}
$$

has simple elementary divisors and real eigenvalues $\lambda$.

We impose more severe conditions on the matrices $A^{\mu}$. We require that each of the matrices $A^{\mu}$ be symmetric and that there exist a particular set $\xi^{0}$ such that the matrix

$$
\sum_{\mu} \xi_{\mu}^{0} A^{\mu}
$$

is positive-definite. If these conditions are satisfied we call the system "symmetric hyperbolic." Obviously, such a system is hyperbolic in the sense of Petrovskii. 
One easily verifies that the initial problem of a hyperbolic equation of the second order

$$
\sum_{\mu, v=1}^{m} a^{\mu \nu} D_{\mu} D_{\nu} u=f
$$

for a single function $u$ is equivalent with the initial problem for a symmetric system. Without restriction we may assume that the $x_{m}$-direction is time-like and that the hyperplanes $x_{m}=$ constant are space-like. Accordingly, we may assume $a_{m m}>0$ and further that the matrix $a_{\tilde{\mu} \tilde{\nu}}$ is negative-definite. Here the tilde indicates that values $m$ of the subscript should be omitted. We then introduce the system

$$
\hat{u}=\left\{u_{1}, \cdots, u_{m}, u\right\}
$$

and set up for it the equations

$$
\begin{gathered}
-\sum_{\tilde{\mu}} a_{\tilde{\mu} \tilde{\nu}} D_{m} u_{\tilde{\mu}}+\sum_{\tilde{\mu}, \bar{\nu}} a_{\tilde{\mu} \tilde{\nu}} D_{\tilde{\mu}} u_{m}=0, \\
\sum_{\tilde{\mu}, \tilde{\nu}} a_{\tilde{\mu} \tilde{\nu}} D_{\tilde{\mu}} u_{\tilde{\nu}}+\left(2 \sum_{\tilde{\mu}} a_{\tilde{\mu} m} D_{\tilde{\mu}}+a_{m m} D_{m}\right) u_{m}=f, \\
D_{m} u-u_{m}=0 .
\end{gathered}
$$

Evidently this system satisfies the requirements formulated.

The initial data should be so chosen that $u_{\tilde{\mu}}=D_{\tilde{\mu}} u$. Since for a solution of the new problem the relation

$$
D_{m} u_{\tilde{\mu}}=D_{\tilde{\mu}} u_{m}=D_{\tilde{\mu}} D_{m} u=D_{m} D_{\tilde{\mu}} u
$$

holds, the identity $u_{\tilde{\mu}}=D_{\tilde{\mu}} u$ is valid, throughout, since it is valid initially.

From the theorems which will be derived for symmetric hyperbolic systems one naturally could derive theorems about equations of second order for one function. However, we shall not do this but confine ourselves to treating only symmetric hyperbolic systems of the first order.

Actually we shall impose two further conditions on the matrices $A^{\mu}$, namely that all of them be non-negative symmetric and that the sum $\sum_{\mu} A^{\mu}$ be positive definite. This is no restriction of generality. One can easily achieve that this condition is satisfied in the neighborhood of any point, e.g. by introducing $y^{\mu}=\sum_{\rho} \xi_{\rho}^{0} x^{\rho}-\epsilon \xi_{\mu}^{0} x^{\mu}$ as new independent variables. Assuming $A^{\mu}$ to be nonnegative and $\sum_{\mu} A^{\mu}$ to be positive definite we shall in the following denote the independent variables by

$$
y=y^{1}, \cdots, y^{m},
$$

but otherwise retain the original notations.

The domain $Q$ in the $y$-space in which the solution of the equation will be sought is bounded by an initial surface $S$ and an end surface $J$. It is an essential requirement of our approach that one should be permitted to interchange the roles of these two surfaces. For this reason we assume the region $a$ to be "lens- 
shaped". Specifically, we assume that it should be possible to cover the region Q by a set of segments parallel to the $u_{\mu}$-axis, for each $\mu, \mu=1, \cdots, m$. We set $d x^{1} \cdots d x^{m}=d x$ in $\mathbb{R}$ and $d x^{1} \cdots d x^{m} / d x^{\mu}=d x_{\mu}^{*}$ on $\mathcal{S}$ and J. Automatically, the surfaces $\delta$ and $\Xi$ are space-like.

Having made these assumptions we can write the energy integral identity in the form

$$
\int_{3} \sum_{\mu}\left(u, A^{\mu} u\right) d x_{\mu}^{*}=2 \int_{\mathcal{G}}(u, E u) d x+\int_{\Omega}(u, J u) d x+\int_{s} \sum_{\mu}\left(u, A^{\mu} u\right) d x_{\mu}^{*},
$$

where the abbreviation $(u, v)$ is used for the "local" inner product,

$$
(u, v)=u_{1} v_{1}+\cdots+u_{p} v_{p} .
$$

The matrix $J$ depends on $B$ and the derivatives of $A^{1}, \cdots, A^{m}$.

Let $u$ be a solution of the equation $E u=0$ which vanishes on $\mathcal{S}$. Suppose first $J=0$. Then we may conclude that $u$ vanishes also on 3 . To this end one could sweep out the region $R$ by a set of lenses $R_{\varepsilon}, 0 \leq \epsilon \leq 1$, with $S_{\epsilon}$ on $S$ and $J_{1}=J$ and then apply the statement made to each region $\mathbb{R}_{\epsilon}$. Instead we shall employ for this purpose minor modifications of the energy integral identity in order to conclude that $u$ vanishes in the whole region $R$, even if $J \neq 0$. In either way, the uniqueness of the solution of the equation $E u=f$ with given values on $S$ can be proved.

So far we have not specified the class of functions $u(y)$ admitted for consideration. First we require that $u$, and also $f$, be quadratically integrable over a. In order that the differential operator $E$ be applicable on the function $u$, we do not require that the operator $E$ be applicable in the "strict" sense, i.e. that $u$ possess continuous derivatives. Instead, we use two extensions of the notion of differential operator.

We first introduce the adjoint $E^{*}$ of the operator $E$ in such a way that the "adjointness" identity

$$
\int_{\mathfrak{Q}}\left(E^{*} v, u\right) d x=\int_{\mathfrak{Q}}(v, f) d x+\int_{\mathbb{S}} \sum_{\mu}\left(v, A^{\mu} g\right) d x_{\mu}^{*},
$$

the analogue of Green's formula, holds for all continuously differentiable functions $v$ which vanish on 5 , provided $u$ satisfies the equation $E u=f$ in $R$ with $u=g$ on $\delta$ in the strict sense. If to any function $u$ two functions $f$ and $g$ exist such that this identity holds for every funetion $v$ with the described property, we say that $u$ admits the operator $E$ in the "weak" sense. We then set $E u=f$ in $R, u=g$ on $\delta$ by definition.

Similar weak definitions of differential operators, in particular of hyperbolic differential operators, have frequently been employed, see e.g. [7A], [12]. The extension of differentiation introduced by L. Schwartz is related to this weak extension, but it differs from it, inasmuch as for the extension of Schwartz, derivatives are not required to exist either as quadratically integrable functions or as elements of a normed space. 
We shall say that the function $u$ admits the operator $E$ in the "strong" sense if functions $f$ and $g$ and a sequence of continuously differentiable functions $u^{(\sigma)}$ exist such that, as $\sigma \rightarrow \infty$,

$$
\begin{gathered}
\int_{\Omega}\left|u^{(\sigma)}-u\right|^{2} d x \rightarrow 0 \\
\int_{\mathscr{Q}}\left|E u^{(\sigma)}-f\right|^{2} d x \rightarrow 0, \quad \int_{\delta}\left|u^{(\sigma)}-g\right|^{2} d x / d x_{\mu} \rightarrow 0 .
\end{gathered}
$$

Again, we set $E u=f$ in $R$ and $u=g$ on $\$$.

Clearly, the uniqueness theorem indicated above holds also if the operator $E$ entering it is understood in the strong sense.

It is remarkable that the solution $u$ of the equation $E u=f, u=g$ on $s$ is unique even if the operator $E$ involved is understood in the weak sense.

This fact is closely related to the fact that the strong and the weak extensions of the operator $E$ agree.

We shall use the uniqueness of the weak solution in proving the existence in the region $R$ of the strong solution ${ }^{3}$ of the equation $E u=f$ with $u=g$ on $S$. In doing this we shall make use of a basic fact of the theory of Hilbert Space.

For simplicity we assume $g=0$. Let the function $v(y)$ be orthogonal to all those functions $f(y)$, called "special," for which there exists a solution $u(y)$ of the equation $E u=f$ with $u=0$ on $\mathrm{S}$. Then $v$ satisfies the equation

$$
E^{*} v=0 \text { in } R \text {, with } v=0 \text { on } \mathfrak{J}
$$

in the weak sense. The theorem of the uniqueness of the weak solution, applied to $v$ with $E^{*}$ instead of $E$, yields $v=0$ identically in $a$. From the theory of the Hilbert space it then follows that the manifold of special functions $f$ covers the whole space of quadratically integrable functions. Thus it is seen that the equation $E u=f$ possesses a solution for an arbitrary function of this kind.

The existence theorem proved in this manner guarantees that a solution, given on a space-like surface $\mathcal{S}$, can be continued into a neighborhood $R$, provided this neighborhood is bounded by a space-like surface $J$, in addition to $\mathcal{S}$. No difficulty is anticipated in proving the existence of the solution in the full domain of determinacy of $S$ bounded by a characteristic surface $J$; but this proof will not be given here.

The arguments outlined will be carried out in detail in Part I of this paper.

Part II contains a proof of the differentiability theorem, which states that the solution of the equation $E u=f$ with $u=g$ on $\$$ admits all individual operators $D_{1}, \cdots, D_{m}$ if this is the case of the right members $f$ and $g$, provided the matrices $A^{\mu}$ and $B$ have continuous derivatives.

\footnotetext{
${ }^{3}$ Thus we derive the existence of the strong solution of an equation from the uniqueness of the weak solution of the adjoint equation. In a certain sense this procedure is the opposite of that by Holmgren [14], who derives the uniqueness of the solution of an analytic differential equation from the existence of the solution of the adjoint equation.
} 
With the aid of arguments in which the identity of the weak and the strong extension is conveniently used we can reduce this theorem to the special case in which $f=0$ on $\&$ and $g=0$.

In order to prove this special differentiability theorem we shall approximate the solution $u$ by solutions $u_{\lambda}$ of finite difference equations. No difficulty arises in setting up initial values for the solutions $u_{\lambda}$, since the initial values of $u$ and $E u$ vanish.

It turns out that the simplest way of setting up difference equations is perfectly sufficient to insure the existence of appropriate limits if one lets the mesh width $\lambda$ approach zero. Denoting by $[\mu] y$ the point

$$
[\mu]\left\{y_{1}, \cdots, y_{\mu}, \cdots, y_{m}\right\}=\left\{y_{1}, \cdots, y_{\mu}+\lambda, \cdots, y_{m}\right\},
$$

we simply replace the equation

$$
\sum_{\mu} A^{\mu}(y) D_{\mu} u(y)=f(y)
$$

by the equation

$$
\sum_{\mu} A^{\mu}(y) u(y)-\sum_{\mu} A^{\mu}(y) u([\mu] y)=\lambda f(y) .
$$

Although the energy integral identity appears to have no analogue which involves only positive definite terms, there is an analogue to the energy integral inequalities derived from it.

The existence of the solution of the difference equation is obvious, and so is the analogue of the differentiability theorem. The convergence of these solutions of the difference equations to the solution $u$ of the differential equation when the mesh width approaches zero is easily deduced from the energy integral inequalities.

The statement that the solution $u$ admits the operators $D_{\mu}$ in the weak sense is an immediate outcome of the limit process. Since the weak and the strong extension of these operators coincide, the statement of the differentiability theorem ensues.

It would seem likely that arguments similar to those employed by Schauder and Frankl could be developed to prove the unique existence of the solution of a quasilinear symmetric hyperbolic equation. However, this will not be done in this paper.

\section{PART I. EXISTENCE AND UNIQUENESS}

\section{Domain and Functions}

We shall consider a space of $m$ independent variables $y^{1}, \cdots, y^{m}$ or points $y=\left\{y^{1}, \cdots, y^{m}\right\}$; the set of variables $y^{1}, \cdots, y^{m}$ from which $y^{r}$ is omitted will be denoted by $y_{r}^{*}$. In the space of points $y$ we consider a "lens-shaped" region $\mathcal{R}^{\prime}$ which can be described as follows. 
We assume that to every value of $r$ for $r=1, \cdots, m$, a pair of continuous functions, $s^{r}\left(y_{r}^{*}\right)$ and $t^{r}\left(y_{r}^{*}\right)$, is given such that each of the conditions

$$
s^{r}\left(y_{r}^{*}\right)<y^{r}<t^{r}\left(y_{r}^{*}\right)
$$

describes the same region $\mathbb{R}^{\prime}$ in the $y$-space; we also require that this domain be bounded.

Of course, we could have described the domain by any one of these inequalities together with appropriate conditions on $s$ and $t$; we found it preferable, however, not to favor a particular variable $y_{r}$.

We further assume that each of the $m$ conditions

$$
s^{\tau}\left(y_{\tau}^{*}\right)=y^{r}<t^{\tau}\left(y_{r}^{*}\right)
$$

describes the same surface, denoted by $s^{\prime}$, and that each of the conditions

$$
s^{r}\left(y_{r}^{*}\right)<y^{r}=t^{r}\left(y_{r}^{*}\right)
$$

describes the same surface, denoted by $\Im^{\prime}$. Evidently, $\mathfrak{S}^{\prime}$ and $\Im^{\prime}$ lie on the boundary of the domain $R^{\prime}$. The remainder of the boundary of $R^{\prime}$ may be visualized as the "edge" of the lens. An additional condition will be formulated in Section 3.

The closures of the open sets $\mathbb{R}^{\prime}, S^{\prime}, J^{\prime}$ will be denoted by

$$
\overline{\mathfrak{R}^{\prime}}=\mathfrak{R}, \quad \overline{\mathfrak{S}^{\prime}}=\mathfrak{s}, \quad \overline{\mathfrak{J}^{\prime}}=\mathfrak{J} .
$$

The surface $S$ will carry the initial values of the solution of the differential equation to be considered. The reason for assuming the domain $R^{\prime}$ to be lensshaped is that our method depends essentially on the possibility of interchanging the roles of $\delta$ and $\zeta$ as initial and end surface. Contrary to custom we have not employed a description of the domain in which a particular variable is distinguished as the "time"; we found it convenient to have the possibility to let any one of the $m$ variables assume the role of the time if this is opportune.

On occasion we shall use the domain $\Omega_{r}^{\prime}$ of all points $y$ in $\mathbb{R}^{\prime}$ which satisfy each of the conditions (1.1)

$$
s^{r}\left(y_{\tau}^{*}\right)<y^{r}<t^{r}\left(y_{r}^{*}\right)-\tau
$$

for any number $\tau \geq 0$. In fact, this domain is of the same type as the domain $\mathfrak{R}^{\prime}$. One need only replace the function $t^{r}\left(y_{r}^{*}\right)$ in (1.1) by the function

$$
t_{\tau}^{r}\left(y_{r}^{*}\right)=\min _{\rho \neq-}\left\{t^{r}\left(y_{r \rho}^{* *}, y^{\rho}+\tau\right), t^{r}\left(y_{r}^{*}\right)-\tau\right\} ;
$$

here $y_{r}^{* *}$ consists of the $m-2$ numbers obtained from $y^{1}, \cdots, y^{m}$ by omitting $y^{r}$ and $y^{p}$. The surfaces $\delta_{\tau}^{\prime}, \mathfrak{J}_{\tau}^{\prime}$ and the closures $\mathbb{R}_{\tau}, \mathfrak{S}_{\tau}, \mathfrak{J}_{\tau}$ can then be introduced accordingly.

We consider vectors $u=\left\{u_{1}, u_{2}, \cdots\right\}, v=\left\{v_{1}, v_{2}, \cdots\right\}, w=\left\{w_{1}, w_{2}, \cdots\right\}$ with the inner product

$$
(u, v)=u_{1} v_{1}+u_{2} v_{2}+\cdots,
$$


and the absolute value $|u|=(u, u)^{1 / 2}$. We need not specify the number of components of these vectors but we assume it to be finite. This assumption is not essential, however; the vectors could as well be assumed to be elements of a Hilbert space, since only slight, and rather obvious, changes of the wording would be necessary to include such a generalization.

We then consider functions $u(y), v(y), \cdots$, which assign vectors $u, v, \cdots$ to every point $y$ in the closed domain $\Omega$; the functions $u(y), v(y), \cdots$ could also be considered as systems of scalar functions. We introduce the linear space $\sqrt{ }$ of all continuous functions defined in $R$ and the space $\mathbb{V}_{1}$ of all functions in (5 possessing continuous derivatives in $R$. The derivatives at boundary points of $R$ are, of course, understood as one-sided derivatives.

We shall also consider functions $\tilde{u}, \tilde{b}, \cdots$ of a point which ranges over $s$ or 3. These functions may be considered as functions of $m-1$ variables, namely, of any of the sets $y_{1}^{*}, \cdots$, or $y_{m}^{*}$. We assume that the functions $\tilde{u}, \tilde{v}, \cdots$ are such that each of the $m$ corresponding functions of $y_{1}^{*}, \cdots$, or $y_{m}^{*}$ is continuous. We simply write $\tilde{u}=\tilde{u}\left(y^{*}\right), \tilde{v}=\tilde{v}\left(y^{*}\right), \cdots$ without specifying which of the $m$ sets of $m-1$ variables is to be taken. The classes of these functions will be denoted by $\mathbb{C}_{s}$ and $\mathfrak{C}_{5}$ respectively.

In particular, such functions $\widetilde{u}\left(y^{*}\right)$ result as the "values" on $\S$ and $\Im$ of functions $u(y)$ in $\mathbb{S}$ when the argument $y$ is specified to represent a point on $\delta$ or 3 respectively. That is, these boundary values are obtained by replacing one of the variables $y^{r}$ by $s^{r}\left(y_{r}^{*}\right)$ or $t^{r}\left(y_{r}^{*}\right)$. It is convenient to use an operator notation for this operation of taking the "value" of $u(y)$ on $s$ and 7 ; we denote these operators by $S$ and $T$ and write the result of applying the operators $S$ and $T$ on $u(y)$ in the forms $S u\left(y_{r}^{*}\right)$ and $T u\left(y_{r}^{*}\right)$. That is, we write

$$
\begin{aligned}
& S u\left(y_{r}^{*}\right)=u\left(y_{r}^{*}, s^{r}\left(y_{r}^{*}\right)\right), \\
& T u\left(y_{r}^{*}\right)=u\left(y_{r}^{*}, t^{r}\left(y_{r}^{*}\right)\right) .
\end{aligned}
$$

The spaces of all functions $\tilde{u}\left(y^{*}\right)$ which can be obtained as $\tilde{u}=S u\left(y^{*}\right)$ and $\tilde{u}=T u\left(y^{*}\right)$ from a function $u(y)$ in $\mathfrak{E}_{1}$ will be denoted by $S \mathfrak{G}_{1}$ and $T \mathfrak{E}_{1}$ respectively.

\section{Operators}

In the following we distinguish between "matrices," operating on vectors, and (linear) "operators," operating on functions $u(y)$. If a matrix is considered as a function of $y$ it is at the same time an operator.

We shall employ various customary notions concerning matrices. We introduce the modulus $|M|$ of $M$ as the least upper bound of the absolute value $|(v, M u)|$ of the inner product $(v, M u)$ taken for all vectors $u, v$ with $|u|=$ $|v|=1$. If the matrix $M=M(y)$ depends continuously on the point $y$, the same is true of $|M|$. We also introduce the least upper bound of $|M(y)|$ as 
regards $y$ in $\mathcal{R}$,

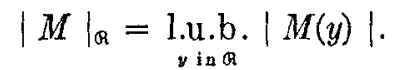

The transpose of a matric $M$ will here be denoted by $M^{*}$, i.e.

$$
(v, M u)=\left(M^{*} v, u\right) .
$$

A matrix $M$ is symmetric if it is equal to its adjoint, $M^{*}=M$; such a matrix is non-negative if $(u, M u) \geq 0$ for all vectors $u$ and positive-definite if equality holds only for $u=0$.

We introduce a set of matrices $A^{r}=A^{r}(y)$ for $r=1, \cdots, m$, defined as continuous functions with continuous deviatives in the closed region $R$. We assume that all matrices $A^{r}$ are symmetric and non-negative; in addition, we assume that $\sum_{\rho} A^{\rho}=\sum_{\rho=1}^{m} A^{\rho}$ is positive definite. Specifically, we assume that there is a positive constant $a$ such that the inequality

$$
\left(u, \sum_{\rho} A^{p} u\right) \geq a(u, u) \text { in } \mathfrak{R}
$$

holds. Any such system of matrices $A^{r}$ will be referred to as a system of type (A).

By $D_{r}$ we denote differentiation with respect to $y^{r}$, i.e. we write

$$
D_{r} u=\partial u / \partial y^{r} \quad \text { for } \quad u \text { in } \mathfrak{c}_{1}, \quad r=1, \cdots, m .
$$

With the aid of the operators $D_{r}$ we then introduce the "basic" symmetric hyperbolic differential operator

$$
A^{\rho} D_{\rho}=\sum_{\rho=1}^{m} A^{\rho} D_{\rho}
$$

which transforms any function $u(y)$ in $\mathcal{C}_{1}$ into the function $A^{\rho} D_{\rho} u(y)$ in $(\mathbb{E}$. The usual summation convention is used here and in the following for summation from 1 to $m$ with respect to Greek indices appearing as sub and superscripts.

We further introduce matrices (not necessarily symmetric) which as functions of $y$ are continuous in $R$. We then also speak of a matrix of type $(B)$. We employ one such matrix $B$ to form the operator

$$
E=A^{\rho} D_{\rho}+B \text {. }
$$

This operator $E$ is the subject of the present investigation.

Any operator of the form $E$ with matrices $A^{r}$ and $B$ as described will be called an operator of type $(E)$. Each of the operators $D_{l}$, for example, is of type $(E)$, since the matrices $A^{r}=0$ for $r \neq l, A^{l}=1, B=0$ satisfy the conditions formulated.

We assign to the operator $E$ its "formal-adjoint" $E^{*}$ given by the expression

$$
E^{*}=-D_{\rho} A^{\rho}+B^{*}
$$


in which $B^{*}$ is the transpose of $B$. The character of $E^{*}$ as the "formal" or "local" adjoint of $E$ is expressed by the relation

$$
(w, E u)-\left(E^{*} w, u\right)=D_{\rho}\left(w, A^{o} u\right),
$$

which holds for any functions $u$ and $w$ in $\mathbb{S}_{1}$.

We denote by $D_{r} A^{8}$. the matrix which is obtained by differentiation of the elements of the matrix $A^{s}(y)$ with respect to $y^{r}$. The operator $D_{r} A^{s}$, without dot, is not a matrix; to apply it on a function $u(y)$ one should first apply the matrix $A^{s}(y)$ and then differentiate the result, $A^{s}(y) u(y)$, with respect to $y$. Evidently, the identity

$$
D_{r} A^{s}=A^{s} D_{r}+D_{r} A^{s}
$$

holds. Using it, we may write the operator $E^{*}$ in the form

$$
E^{*}=-A^{\rho} D_{\mathrm{\rho}}-D_{\mathrm{p}} A^{\rho} \cdot+B^{*},
$$

which shows that $-E^{*}$ is an operator of type $(E)$.

\section{Bilinear Forms; Adjointness Formula}

We use the abbreviations

$$
\begin{aligned}
& \int \cdots d y=\int \cdots \int \cdots d y^{1} \cdots d y^{m} \\
& \int \cdots d y_{r}^{*}=\int \cdots \int d y^{1} \cdots d y^{m} / d y^{r} .
\end{aligned}
$$

Integrations over the open domains $R^{\prime}, S^{\prime}, J^{\prime}$ will respectively be denoted by

$$
\int_{\mathscr{R}} \cdots d y, \quad \int_{s} \cdots d y_{r}^{*}, \quad \int_{s} \cdots d y_{r}^{*} .
$$

For functions $u, w$ in the space $\mathbb{E}$ we introduce the bilinear form

$$
(w, u)_{\mathfrak{Q}}=\int_{\mathfrak{R}}(w, u) d y .
$$

We now formulate the additional condition to be imposed on the surfaces $\S$ and $\mathfrak{I}$ referred to in Section 1 . There should be positive numbers $\sigma_{r}$ defined as continuous functions of the points of $\delta$ such that the ratio $d y_{r}^{*} / \sigma_{r}$ is independent of $r$,

$$
d y_{r}^{*} / \sigma_{r}=d y_{i}^{*} / \sigma_{l} .
$$

If the surface $S$ possesses a continuous normal, which we might just as well have assumed, the components of this normal could be taken as the numbers $\sigma_{+}$.

We make this assumption $\left(\sigma_{r}\right)$ for convenience although the results of Part I could be obtained independently of it. 
For functions $\tilde{u}, w$ in $\widehat{S}_{s}$ we then set

$$
(\tilde{w}, \tilde{u})_{s}=\int_{\Omega}(\tilde{w}, \tilde{u}) d y_{r}^{*} / \sigma_{r}
$$

and note that this expression is independent of $r$.

Similarly, we make the assumption $\left(\tau_{r}\right)$ that continuous positive functions $\tau_{r}$ can be introduced on the surface $J$, such that the ratio $d y_{r}^{*} / \tau_{r}$ is independent of $r$,

$$
d y_{\tau}^{*} / \tau_{r}=d y_{i}^{*} / \tau_{l}
$$

We then set

$$
(\tilde{w}, \tilde{u})_{3}=\int_{\Im}(\tilde{w}, \tilde{u}) d y_{r}^{*} / \tau_{r}
$$

for functions $\tilde{u}, \tilde{w}$ in $\widetilde{E}_{3}$.

In general we shall let the functions $\tilde{u}, \tilde{w}$ belong to the classes $S \mathscr{C}_{1}$ or $T \mathscr{C}_{1}$; i.e. let them be boundary values of functions in $\mathcal{G}_{1}$ defined in $\mathcal{R}$. If functions

$$
\tilde{u}=S u \quad \text { or } \quad \tilde{u}=T u
$$

of this type enter any of the bilinear forms we shall in general omit the operator sign $S$ or $T$, i.e. we shall write

$$
(\tilde{w}, S u)_{s}=(\tilde{w}, u)_{s},
$$

and

$$
(\tilde{w}, T u)_{\Im}=(\tilde{w}, u)_{\jmath}
$$

Similarly, we shall omit $S$ or $T$ if $w$ is expressed as $S w$ or $T w$.

For functions $u, w$ in $\varsigma_{1}$ we evidently have the identity

$$
\int_{\mathfrak{Q}} D_{r}(w, u) d y=\int_{\mathrm{J}}(w, u) d y_{\mathrm{r}}^{*}-\int_{\delta}(w, u) d y_{r}^{*}
$$

whence, using the definitions (3.3),

$$
\int_{\mathscr{R}} D_{\mathrm{r}}(w, u) d y=\left(w, \tau_{\mathrm{r}} u\right)_{\Im}-\left(w, \sigma_{\tau} u\right)_{\S} .
$$

Similarly, we have

$$
\int_{\mathscr{R}} D_{r}\left(w, A^{r} u\right) d y=\left(w, A^{r} \tau_{\tau} u\right)_{\Im}-\left(w, A^{r} \sigma_{\tau} u\right)_{s} .
$$

By integrating identity (2.8) over $\mathcal{R}^{\prime}$ we therefore obtain

Theorem 3: The identity

$$
(w, E u)_{\Re}-\left(E^{*} w, u\right)_{\Re}+\left(w, A^{p} \sigma_{\rho} u\right)_{s}-\left(w, A^{p} \tau_{\rho} u\right)_{3}=0
$$

is valid for functions $u$ and $w$ in $\mathfrak{S}_{1}$. 
Identity (3.6), which corresponds to "Green's formula", expresses the relationship between the adjoint operators $E$ and $E^{*}$ in terms of the bilinear forms; we shall refer to it as the "adjointness formula."

Clearly, the quantities $A^{\rho} \sigma_{\rho}$ and $A^{\rho} \tau_{\rho}$, which occur in the adjointness formula, are non-negative on $\$$ and $\Im$. This fact implies that these surfaces are spacelike.

We further note that the combinations

$$
\sigma_{\tau} S D_{k}-\sigma_{k} S D_{r}, \quad \tau_{r} T D_{k}-\tau_{k} T D_{r}
$$

involve differentiation in a direction within the surfaces $\$$ and 3 .

\section{Hilbert Spaces}

Evidently, the quadratic form $(u, u)_{\mathbb{N}}$, given by (3.1), does not vanish for functions $u(y) \neq 0$ in $\mathfrak{S}$. Also the quadratic forms $(\tilde{u}, \tilde{u})_{s}$ and $(\tilde{u}, \tilde{u})_{s}$, given by (3.3), do not vanish for functions $\tilde{u}(y) \neq 0$ in $\mathfrak{E}_{\mathrm{s}}$ and $\mathfrak{E}_{\mathrm{J}}$, as is readily verified in view of assumption (2.3).

With respect to the norms

$$
\begin{array}{llll}
\|u\|_{a}=(u, u)_{\Omega}^{1 / 2} & \text { for } & u \text { in } & \mathbb{E}, \\
\|\tilde{u}\|_{s}=(\tilde{u}, \tilde{u})_{s}^{1 / 2} & \text { for } & \tilde{u} \text { in } \mathfrak{\Xi}_{s}, \\
\|\tilde{u}\|_{J}=(\tilde{u}, \tilde{u})^{1 / 2} & \text { for } & \tilde{u} \text { in } \mathfrak{S}_{5},
\end{array}
$$

we may, consequently, extend the spaces $\sqrt{ } \sqrt{ }, \mathfrak{V}_{s}, \mathfrak{S}_{s}$ to complete spaces, i.e. to Hilbert spaces, denoted by $\mathfrak{S}_{\mathfrak{c}}, \mathfrak{S}_{s}, \mathfrak{S}_{\mathfrak{s}}$ respectively.

Whether this completing process is performed by adjoining certain Lebesguemeasurable functions or by adjoining ideal elements is irrelevant for our investigation. The elements of these three Hilbert spaces are again called functions and again denoted by $u(y)$ and $\tilde{u}\left(y^{*}\right)$.

The following approximation properties of the spaces $\mathfrak{S}_{3}, \mathfrak{S}_{3}$, and $\mathfrak{S}_{3}$ are easily proved; they involve the operators $S$ and $T$ defined by (1.5).

Lemma 4: The space of all functions $u$ in $\mathfrak{S}_{1}$ with $S u=0$ and $T u=0$ is dense in $\mathfrak{5}$ with respect to the norm \|\|$_{G}$.

LEMMA $4_{s}\left(4_{\jmath}\right)$ : The space of all functions $S u(T u)$ resulting from functions $u$ in $\mathfrak{S}_{1}$ with $T u=0(S u=0)$ is dense in $\mathfrak{S}_{s}\left(\mathfrak{S}_{5}\right)$ with respect to the norm \|\|$_{s}$ $\left(\|\|_{3}\right)$.

\section{Extension of the Differential Operator}

We proceed to introduce subspaces of the Hilbert space $\mathfrak{S}$ which contain $\mathfrak{S}_{1}$ and to define in them closed operators which are extensions of the operator $E$ defined in $\mathbb{E}_{1}$. We shall set up a "weak" and a "strong" extension of $E$. Eventually it will be shown that these two extensions coincide. 
Weak and strong extensions of differential operators with constant coefficients and the proof of their identity are treated in various papers [7], [12]. Also the identity of both extensions of differential operators of first order with non-constant coefficients was proved in an earlier paper [12B]. For the present purpose we need slightly modified versions of these extensions; accordingly, the proof of the identity of the different extensions must be adapted to these modifications. For the sake of completeness we shall present certain details which could have been taken over from the earlier work without modification.

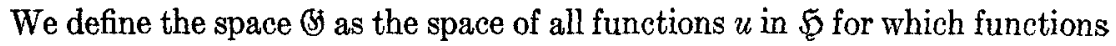
$f$ in $\mathfrak{S}, \tilde{g}$ in $\mathfrak{S}_{s}$, and $\tilde{h}$ in $\mathfrak{S}_{\mathcal{J}}$ exist such that the relation

$$
\left(D^{\rho} A_{\rho} w, u\right)_{\mathscr{Q}}+(w, f)_{\Omega}+\left(w, A^{\rho} \sigma_{\rho} \tilde{g}\right)_{s}-\left(w, A^{\rho} \tau_{p} \tilde{h}\right)_{S}=0
$$

holds for all functions $w(y)$ in $\mho_{1}$.

We then show : if $u=0$, also $f=0, \tilde{g}=0, \tilde{h}=0$. To this end we first restrict the function $w$ by requiring $S w=0$ and $T w=0$ so that relation (5.1) reduces to $(w, f)_{a}=0$. As seen from Lemma 4 , the function $f$ can be approximated with reference to the norm $\|\quad\|_{a}$ by functions $\dot{f}$ in $\xi_{1}$ with $S \dot{f}=T \dot{f}=0$. Clearly $(\dot{f}, f)_{\mathbb{R}}=0$ entails $(f, f)_{A}=0$ and thus $f=0$ follows. We now restrict $w$ only by the condition $T w=0$; then $\left(w, A^{\rho} \sigma_{\rho} \tilde{g}\right)=0$ remains. By virtue of Lemma $4_{s}$, the function $\tilde{g}$ can be approximated with reference to \|\|$_{s}$ by functions $S \dot{g}$ with $\dot{g}$ in $\Theta_{1}$ and $T \dot{g}=0$. Clearly, $\left(\dot{g}, A^{\rho} \sigma_{\rho} \tilde{g}\right)=0$ entails $\left(\tilde{g}, A^{\rho} \sigma_{\rho} \tilde{g}\right)$ $=0$ and thus $\tilde{g}=0$ follows. In the same way $\tilde{h}=0$ is derived.

The fact thus proved guarantees that the functions $f, \tilde{g}, \tilde{h}$ are uniquely and linearly determined by $u$. Consequently, for functions $u$ in (S) three linear operators, denoted by $A D, S$, and $T$, are defined by

$$
A D u=f, \quad S u=\tilde{g}, \quad T u=\tilde{h} .
$$

If the function $u$ lies in $\mathbb{S}_{1}$ it also lies in $\$$, and the operators just defined coincide with those originally denoted by $A^{p} D_{p}, S$, and $T$, respectively, as is easily verified. The new operators will be called the "weak" extensions of the original operators acting in $\mathscr{E}_{1}$.

We observe that the matrix $B$ considered as a linear operator acting on functions $u(y)$ in $\subseteq$ is bounded with respect to \|\|$_{a}$; therefore it can be extended to a bounded operator $B$ in $\mathfrak{Q}$ which transforms "functions" $u(y)$ in $\mathfrak{S}$ into "functions" $B(y) u(y)$ in $\mathfrak{5}$. The same remark applies to any matrix of the type $(B)$ such as $B^{*}$ and $D_{p} A^{p}$. Using the bounded operators we can define the operators

$$
E=A D+B
$$

and

$$
E^{*}=-A D-D_{\rho} A^{\rho} \cdot+B^{*}
$$

acting on functions $u(y)$ in $\$$, cf. (2.6), (2.7). These operators $E$ and $E^{*}$ are then extensions, the "weak" extensions, of the operators $E$ and $E^{*}$ originally defined in $G_{1}$. 
As a consequence of the definition of the extended operator $E, S$, and $T$ the adjointness formula (3.6) holds also for functions $u$ in (5). Of course, our convention of omitting the operator signs $S$ and $T$ in the boundary forms has been retained.

We now turn to the "strong" extension produced by a process of closure. We define the $\mathfrak{F}=\mathfrak{F}(A D)$ as the manifold of all functions $u$ in $\mathfrak{S}$ to which functions $f$ in $\mathfrak{g}, \tilde{g}$ in $\mathfrak{S}_{S}, \tilde{h}$ in $\mathfrak{S}_{\mathfrak{S}}$, and a sequence $u_{\mathfrak{\epsilon}}$ of functions in $\mathfrak{S}_{1}$ exist such that, as $\epsilon \rightarrow 0$

$$
\begin{array}{ll}
\left\|u_{\epsilon}-u\right\|_{Q} \rightarrow 0, & \left\|A^{\rho} D_{\rho} u_{\epsilon}-f\right\|_{R} \rightarrow 0, \\
\left\|u_{\epsilon}-\tilde{g}\right\|_{\delta} \rightarrow 0, & \left\|u_{\epsilon}-\tilde{h}\right\|_{\delta} \rightarrow 0 .
\end{array}
$$

From relation (3.6) applied to $u=u_{\mathrm{s}}$ we immediately infer that relation (4.1) holds for $u$ in $\tilde{F}$. Hence the function $u$ in $\mathfrak{F}$ is also in $\mathfrak{G}$ and $A D u=f$, $S u=\tilde{g}, T u=\tilde{h}$. Thus the operators $A D, S, T$ and also $E^{*}, E^{*}$ are defined in t.

It is remarkable that also the converse holds: every function $u$ in (5) also belongs to $\tilde{F}$; we formulate this fact as the

IDENTIFICATION Theorem $5:(\$)=F$.

It states that the weak and the strong extensions of the operator $A^{\circ} D_{\rho}$ coincide. At the end of Section 8 its proof will be reduced to other statements proved in Part $\mathrm{I}$.

\section{Initial Value Problem}

We are now in a position to formulate the initial value problem. It consists in prescribing functions $f$ in $\mathfrak{S}$ and $\tilde{g}$ in $\mathfrak{S}_{\mathfrak{s}}$, and asking for a function $u$ in $\tilde{F}$ such that the differential equation

$$
E u=f
$$

and the initial condition

$$
S u=\tilde{g}
$$

are satisfied. The main theorem of Part $I$ is that this initial value problem has one and only one solution. We split this statement into a uniqueness and an existience theorem:

UNIQUeness Theorem $6_{s}^{U}$. Suppose $E u=0$ and $S u=0$ for a function in $\mathfrak{F}$. Then $u=0$.

Existence Theorem $6_{s}^{E}$ : Let $f$ be any function in $\mathfrak{S}$ and $\tilde{g}$ be any function in $\mathfrak{S}_{\mathrm{s}}$. Then a function $u$ in $\mathfrak{F}$ exists such that $E u=f S u=g$.

Corresponding theorems $6_{J}^{U}$ and $6_{\jmath}^{E}$ hold. 


\section{Energy Integral Inequality}

The uniqueness theorem will be a consequence of the "energy integral inequality" contained in the

INEQUALITY ThEOREM $7_{\mathrm{s}}$ : A number $\gamma>0$ exists such that for all functions $u$ in $\mathfrak{F}$ the relation

$$
\|u\|_{Q}^{2}+\|u\|_{s}^{2} \leq \gamma^{2}\|E u\|_{Q}^{2}+\gamma^{2}\|u\|_{s}^{2}
$$

holds.

Upon interchanging the roles of $\delta$ and $\mathfrak{J}$ we obtain the formulation of an inequality $(7.1)_{\mathrm{J}}$.

In order to prove inequality $(7.1)_{8}$ we first assume that the function $u$ lies in the space $\mathfrak{C}_{1}$. We then introduce the function

$$
p(y)=\exp \left\{-\theta \sum_{\rho} y^{p}\right\}
$$

in which $\theta$ is a positive number at our disposal, and insert the function

$$
w=p u
$$

in the adjointness formula (3.6). Evidently, we have

$$
A^{\rho} D_{\rho} p u=p\left[A^{\rho} D_{\rho} u-\theta \sum_{\rho} A^{p} u\right],
$$

and hence

$$
E p u=p\left[E u-\theta \sum_{p} A^{p} u\right]
$$

Next we conclude from formula (2.10) that the operator $E^{*}$ can be written in the form

with

$$
E^{*}=-E+C
$$

$$
C=B-B^{*}-D_{\rho} A^{\circ} .
$$

Combining relation $E^{*} u=-E u+C u$ with relation (7.3) we find

$$
E^{*} p u=-p\left[E-C-\theta \sum A^{p}\right] u .
$$

Insertion of this expression with $w=p u$ into formula (3.6) and a slight rearrangement of the resulting terms yields the relation

$$
\left(p u, \theta \sum_{\rho} A^{p} u\right)_{\mathscr{A}}+\left(p u, A^{p} \tau_{\rho} u\right)_{\mathcal{S}}=2(p u, E u)_{\Re}-(p u, C u)_{\mathbb{R}}+\left(p u, A^{\rho} \sigma_{\rho} u\right)_{s} .
$$

Making use of assumption (2.3) and of Schwarz's inequality we obtain the relation

$$
\theta a(p u, u)_{\mathscr{Q}}+(p u, u)_{\mathcal{S}} \leq(p E u, E u)_{\mathbb{Q}}+(1+c)(p u, u)_{\mathscr{Q}}+(p u, u)_{\Sigma},
$$


in which

$$
c=|C(y)|_{\mathcal{Q}} \leq 2|B|_{\mathcal{Q}}+\left|D_{\rho} A^{\rho} \cdot\right|_{\mathcal{Q}},
$$

cf. (2.1). We now choose the number $\theta$ as

$$
\theta=[2+c] a^{-1}
$$

and obtain the relation

$$
(p u, u)_{\Re}+(p u, u)_{\Im} \leq(p E u, E u)_{\Theta}+(p u, u)_{s} .
$$

Finally, we introduce the number

$$
\gamma=\underset{y, \dot{v} \text { i i } \dot{G}}{\operatorname{loxp}}\left\{\theta \sum_{\rho}\left(y^{\rho}-\bar{y}^{\rho}\right) / 2\right\}
$$

and then obtain inequality (7.1) $)_{8}$ from (7.7).

This inequality has been derived for functions $u$ in $\mathbb{E}_{1}$. However, by virtue of the very definition of the space $₹$ and the operators $E, S, T$ defined in $\mathfrak{F}$, the inequality also holds for functions $u$ in $\mathfrak{F}$.

For a later application we mention

LEMMA $7_{s}^{\omega}$ : Let $\omega(y)$ be a function with continuous derivatives for which $0 \leq \omega \leq 1$ and which vanishes in a neighborhood of $\mathrm{S}$. Then a constant $\mathbf{\Omega}$ exists such that the inequality

$$
(\omega u, u)_{\Im} \leq \gamma^{2}\|E u\|_{\Re}^{2}+\gamma^{2} \Omega\|u\|_{\Re}^{2}
$$

holds for all functions in $\mathfrak{F}$.

We need only replace $p$ by $\omega p$ in the proof of Lemma $7_{s}$ and set $\Omega=\left|A^{\rho} D_{\rho} \omega \cdot\right|_{\uparrow}$; then formula (7.9) follows with the same value of $\gamma$ as before, given by (7.8).

\section{Proof of the Uniqueness, Existence and Identification Theorems}

The Uniqueness Theorem $6_{s}^{U}$ is an immediate consequence of the inequality (7.1) $)_{\text {s }}$ for functions $u$ in $\mathfrak{F}$.

In the proof of the Existence Theorem $6_{s}^{E}$ we shall make use of the fact that the only closed subspace of a Hilbert space which is orthogonal to only the element 0 is the whole Hilbert space itself. Owing to this theorem it is possible to reduce the existence of the solution of an equation to the uniqueness of the solution of the adjoint equation. Specifically, we shall derive Theorem ${ }^{E}{ }_{s}^{E}$ from the following

Unioveness Lemma $8_{3}^{U}$ : Suppose $E^{*} u=0$ and $T u=0$ for a function $u$ in B. Then $u=0$.

If the identity of the strong and weak extension of the operator $E$ and hence of $E^{*}$ were proved, Lemma $8_{3}^{U}$ would follow from the Uniqueness Theorem 
$6_{3}^{U}$. Actually, we shall prove Lemma $8_{3}^{U}$ directly, in Sections 10 to 14 , and derive the Identification Theorem 5 from it, at the end of this section.

We now turn to the reduction of the Existence Theorem $6{ }_{s}^{E}$ to the Uniqueness Lemma $8_{J}^{U}$. We consider the space $\mathfrak{S} \times \mathfrak{S}_{\mathfrak{s}}$ of all pairs $\{f, \tilde{g}\}$ of functions $f$ in $\mathfrak{S}$ and $\tilde{g}$ in $\mathfrak{S}_{s}$, and assign to these pairs the unit form $(f, f)_{R}+(\tilde{g}, \tilde{g})_{s}$. The space $E \mathfrak{F} \times S \mathfrak{F}$ of all pairs $\{E w, S w\}$ resulting from a function $w$ in $\mathfrak{F}$ is evidently a subspace of $\mathfrak{S} \times \mathfrak{S}_{\mathfrak{s}}$. This subspace is closed by virtue of inequality $(7.1)_{\mathrm{J}}$. It is now clear that the Existence Theorem $6_{8}^{E}$ can be formulated as the statement that the space $E \mathfrak{F} \times S \mathfrak{F}$ coincides with the whole space $\mathfrak{T} \times \mathfrak{S}_{s}$.

In order to prove this statement we consider an element $\{u, \tilde{g}\}$ in the space $\mathfrak{S} \times \mathfrak{S}_{s}$ perpendicular to the space $E \mathfrak{F} \times S \mathfrak{F}$; i.e. we assume that the relation

$$
(E w, u)_{\Theta}+(w, \tilde{g})_{8}=0
$$

holds for all funetions $w$ in $\mathfrak{F}$ and hence for all functions $w$ in $\mathbb{E}_{1}$. We may write this relation in the form

$$
\left(D_{\rho} A^{p} w, u\right)_{\mathcal{G}}+(w, f)_{\mathscr{G}}+\left(w, A^{\rho} \sigma_{\rho} g\right)_{s}-\left(w, A^{\rho} \tau_{\rho} h\right)_{\mathcal{J}}=0
$$

with $f=\left(B^{*}-D_{\rho} A^{p} \cdot\right) u$ and $\tilde{h}=0$. We then see that relation (5.1) holds for all $w$ in $\mathfrak{S}_{1}$. It follows that the function $u$ belongs to the space $(\mathfrak{S})$ and that $A D u=\left(B^{*}-D_{\rho} A^{\rho} \cdot\right) u$ or $E^{*} u=0, S u=\tilde{g}$, and $T u=0$, in accordance with (5.2) and (2.7).

Lemma $8_{\jmath}^{U}$ now yields $u=0$. Consequently, $S u=0$ and hence also $\tilde{g}=0$. Thus we have shown that $\{0,0\}$ is the only element in the space $\mathfrak{F} \times \mathfrak{F}_{8}$ which is perpendicular to the space $E \mathfrak{F} \times S \mathfrak{F}$. By virtue of the basic fact of the theory of the Hilbert space mentioned at the beginning of this section, the identity $E \mathfrak{F} \times S \mathfrak{F}=\mathfrak{S} \times \mathfrak{W}_{\mathfrak{S}}$ ensues. Thus Theorem $6_{s}^{\mathbb{E}}$ is reduced to Lemma $8_{\mathfrak{J}}^{U}$. We shall prove Lemma $8_{5}^{U}$ in Sections 10 to 14 .

At present we observe that combined with Theorem $6_{5}^{R}$ this lemma immediately yields a proof of Theorem 5 , which expresses the identity of the weak and the strong extension of the operator $E$.

Let $u$ be a function in $(5)$. According to Theorem $6_{5}^{\mathbb{E}}$ a function $u^{\prime}$ in $\mathfrak{F}$ exists such that $E u^{\prime}=E u$ and $T u^{\prime}=T u$. The function $u-u^{\prime}$ lies in (5) and satisfies the assumptions of Lemma $8_{\mathfrak{3}}^{U}$ for $E$ instead of $E^{*}$. Hence $u^{\prime}-u=0$ follows. Therefore, the function $u$ in $\mathfrak{G}$ is at the same time in $\mathfrak{F}$ and the identity $(\mathfrak{s})=\mathfrak{F}$ follows.

\section{Modified Weak Extension}

For later purposes it is necessary to prove a lemma which is somewhat stronger than the Uniqueness Lemma $8_{5}^{U}$; it refers to an extension of the operator $A^{P} D_{\rho}$ which is somewhat weaker than the extension into (5).

We define $\mathbb{S}_{3}$ as the space of all functions $u$ in $\mathfrak{S}$ to which functions $f$ in $\mathfrak{W}, \tilde{h}$ in $\mathfrak{S}_{\text {f }}$ exist such that the relation

$$
\left(D_{\rho} A^{p} w, u\right)_{\Re}+(w, f)_{\Re}-\left(w, A^{p} \tau_{\rho} \bar{h}\right)_{\mathfrak{s}}=0
$$


holds for all functions $w$ in $\$_{1}$ with $S w=0$.

In the manner used for $B$, one shows that $u=0$ implies $f=0$ and $\tilde{h}=0$, and that therefore linear operators $A D$ and $T$ can be defined by

$$
A D u=f, \quad T u=\tilde{h} .
$$

Operators $E$ and $E^{*}$ can then be introduced by (5.3), (5.3)*.

The operators thus defined in the space $\mathfrak{G}_{3}$ are extensions of the operators $A D, T, E, E^{*}$ in $\mathbb{G}_{1}$. Furthermore we observe: if $u$ is in $\mathbb{S}_{5}$, the relation

$$
\left(E^{*} w, u\right)_{\mathfrak{Q}}-(w, E u)_{\mathfrak{G}}+\left(w, A^{\rho} \tau_{\rho} u\right)_{3}=0
$$

holds for all functions $w^{\prime}$ in $\mathbb{E}_{1}$ with $S w=0$.

Similarly we see that a space $B_{8}$ can be defined through a relation $(9.1)_{s}$. Clearly, operators $A D, S, E, E^{*}$ can be defined in $B_{s}$ and the relation $(9.2)_{s}$ holds.

The analogue of Lemma $8_{5}^{U}$ is the

Modified Uniqueness Lemma $9_{\Im}^{v}:$ Suppose $E^{*} u=0$ and $T u=0$ for $a$ function $u$ in $\mathbb{S}_{\Im}$. Then $u=0$.

We shall start the proof of this lemma in Section 10. Evidently, the space $B{ }_{3}$ contains the space $(S)$ since no boundary values $S u$ are required of $u$ in (S) . For this reason Lemma $8_{3}^{U}$ follows from Lemma $9_{3}^{U}$.

Actually the two spaces $\left(\mathcal{S}_{\mathfrak{J}}\right.$ and $(B)$ are identical and, instead of Theorem 5 , we have the

Modified Identification Theorem $9_{\mathfrak{J}}^{I}\left(9_{\mathfrak{s}}^{I}\right): \quad \mathfrak{G}_{\mathfrak{J}}=\mathfrak{F}\left(\mathfrak{G}_{\mathrm{s}}=\mathfrak{F}\right)$.

This statement follows from Lemma $9_{3}^{U}$ by literally the same arguments by which at the end of Section 8 Theorem 5 was derived from Lemma $8^{U}$ (note that Theorem $6_{3}^{E}$, being a consequence of Lemma $8_{3}^{U}$, is also a consequence of Lemma $\left.9_{5}^{U}\right)$.

\section{Main Lemma}

The modified Uniqueness Lemma $9_{3}^{U}$ will be derived from the

MAIN LEMMA $10_{3}$ : To every function $u$ in $\mathfrak{H}_{3}$ which satisfies the equations $E u=0$ and $T u=0$ there is a sequence of functions $u_{\mathrm{e}}$ in $\mathfrak{S}_{1}$ with $T u_{\mathrm{e}}=0, \epsilon \rightarrow 0$, such that

$$
\begin{gathered}
\left\|u_{\epsilon}-u\right\|_{\Theta} \rightarrow 0, \\
\left\|E u_{\varepsilon}\right\|_{\Theta} \rightarrow 0, \quad \text { as } \quad \epsilon \rightarrow 0 .
\end{gathered}
$$

Since inequality $(7.1)_{\Im}$ may be applied to the functions $u_{\epsilon}$, relation (10.2) leads to relation

$$
\left\|u_{\varepsilon}\right\|_{a} \rightarrow 0 \quad \text { as } \quad \epsilon \rightarrow 0 .
$$


Relation (10.1) then leads to $\|u\|_{\odot}=0$ or $u=0$. Thus Lemma $9_{\Im}^{U}$ for $E$ instead of $E^{*}$ is reduced to the main lemma.

The approximating functions $u_{e}$ in $\mathfrak{S}_{1}$, to be established in proving the main lemma, will be constructed by means of a sequence of certain integral operators, the "mollifiers" $J_{\epsilon}$. For the sake of completeness, and because of minor modifications needed, we shall formulate and derive various properties of integral operators in general and of the mollifiers in particular, although this was essentially done in an earlier work [12B].

\section{Integral Operators}

Let $k(y, \bar{y})$ be a matrix which depends continuously on the pair of points $(y, \bar{y})$ in $\mathbb{R} \times \mathbb{R}$ and is bounded there. This matrix may be taken as the kernel of an integral operator $K$, which transforms the function $u(y)$ in $\mathbb{E}$ into the function

$$
K u(y)=\int_{\Re} k(y, \bar{y}) u(\bar{y}) d \bar{y},
$$

in 5 . Various familiar notions will be employed in connection with such operators. We assign to the operator $K$ as its "norm" the smallest number $\|K\|$ such that the inequalities

$$
\begin{aligned}
& \int_{\Omega}|k(y, \bar{y})| d \tilde{y} \leq\|K\| \quad \text { for } \quad y \text { in } a, \\
& \int_{\Omega}|k(y, \bar{y})| d y \leq\|K\| \quad \text { for } \quad \bar{y} \text { in } a
\end{aligned}
$$

hold. Then the inequality

$$
\|K u\|_{a} \leq\|K\|\|u\|_{a}
$$

holds for functions $u$ in $\mathfrak{S}$. In fact, we have from Schwarz's inequality

$$
|K u(y)|^{2} \leq \int_{\Omega}|k(y, \bar{y}) d \bar{y}| \int_{\Omega}|k(y, \bar{y})||u(\bar{y})|^{2} d \bar{y}
$$

and hence

$$
\leq\|K\| \int_{Q}|k(y, \bar{y})||u(\bar{y})|^{2} d \bar{y}
$$

$$
\begin{aligned}
\int_{a}|K u(y)|^{2} d y & \leq\|K\| \int_{\mathscr{Q}}\left[\int_{\mathscr{a}}|k(y, \bar{y})| d y\right]|u(\bar{y})|^{2} d \bar{y} \\
& \leq\|K\|^{2} \int_{a}|u(\bar{y})|^{2} d \bar{y},
\end{aligned}
$$

whence (11.3). 
Relation (11.3) shows that the operator $K$, so far defined in $\sqrt{5}$, can be extended to the whole space $\mathfrak{S}$ so that $(11.3)$ remains valid. Relation

$$
|K u(y)|^{2} \leq\|K\| \underset{y \text { in } \Omega_{i \text { in } a}}{\text { l.u.b. }}|k(y, \bar{y})|\|u\|^{2},
$$

which follows from (11.4), shows that the function $K u(y)$ lies in 5 when $u$ is in $\mathfrak{S}$. If $k(y, \bar{y})$ possesses continuous derivatives with respect to $y^{r}$, it is clear that the function $K u(y)$ also possesses continuous derivatives.

The adjoint $K^{*}$ of the operator $K$, defined as the integral operator with the kernel $k(\bar{y}, y)$, transforms the function $u(y)$ in 5 into the function

$$
K^{*} u(y)=\int_{\Re} k(\bar{y}, y) u(\bar{y}) d \bar{y}
$$

The operators $K$ and $K^{*}$ are related through the identity

$$
\left(K^{*} w, u\right)_{\mathbb{Q}}=(w, K u)_{\Omega},
$$

which holds for functions $u, w$ in $\mathfrak{S}$.

We now consider a set of integral operators $K$, with the kernels $k_{\epsilon}(y, \bar{y})$ depending on a parameter $\epsilon>0$. We assume that they enjoy the following properties:

P I. There is a number $k_{0} \geq 0$ such that the relation

$$
\left\|K_{\epsilon}\right\| \leq k_{0}
$$

holds for all $\epsilon$.

In other words, the norms of the operators $K_{\varepsilon}$, defined by (11.2), should be uniformly bounded.

P II. There is a number $\mathrm{k}$ and for every $\tau>0$ a number $\epsilon(\tau)>0$ such that

$$
\int_{\mathfrak{a}} k_{e}(y, \bar{y}) d \bar{y}=\kappa
$$

if $y$ lies in the domain $\mathbb{R}_{\tau}$, defined through $(1.1)_{\tau}$, and $\epsilon \leq \epsilon(\tau)$.

$\mathrm{P}$ III. The relation

$$
k_{e}(y, \bar{y})=0
$$

should hold outside of the cube $\left|y^{r}-\bar{y}^{r}\right| \leq 3 \epsilon, r=1, \cdots, m$.

From these three properties we shall deduce that the relation

$$
\left\|K_{\epsilon} u-\kappa u\right\|_{Q} \rightarrow 0 \quad \text { as } \quad \epsilon \rightarrow 0
$$

holds for all functions $u$ in $\$$

Suppose first that the function $u$ is in $\mathbb{S}$. For $y$ in $\mathbb{R}_{\tau}$ and $\epsilon \leq \epsilon(\tau)$ we then may deduce from $(11.7,11.6)$ the relation

$$
\left|K_{\epsilon} u(y)-\kappa u(y)\right|=\left|\int_{\mathfrak{Q}} k_{\epsilon}(y, \bar{y})[u(\bar{y})-u(y)] d \bar{y}\right| \leq k_{0} M_{3 \epsilon}
$$


where $M_{3 \mathrm{e}}$ is the maximum of $|u(y)-u(\bar{y})|$ in the cube $\left|y^{r}-\bar{y}^{r}\right| \leq 3 \epsilon$, $r=1, \cdots, m$. Owing to the uniform continuity of $u(y)$ in $R_{\tau}$, the last member of relation (11.10) tends to zero with $\epsilon$. Hence we have

$$
\int_{\mathscr{R}}\left|K_{\iota} u(y)-\kappa u(y)\right|^{2} d y \rightarrow 0 \quad \text { as } \quad \epsilon \rightarrow 0 .
$$

On applying inequality (11.3) to the domain $R-R_{r}$ instead of $R$ we find

$$
\int_{\mathfrak{A}_{-}-\mathfrak{G}_{\tau}}\left|K_{\epsilon} u(y)\right|^{2} d y \leq k_{0} \int_{\mathfrak{R}_{-\mathfrak{A}_{\tau}}}|u(y)|^{2} d y .
$$

Hence we obtain

$$
\left\|K_{\boldsymbol{\epsilon}} u-u\right\|^{2} \leq \int_{\mathcal{R}_{\tau}}\left|K_{\mathbf{\epsilon}} u-\kappa u\right|^{2} d y+2\left(k_{0}+|\kappa|\right) \int_{\mathbb{R}_{-} \mathcal{G}_{\tau}}|u|^{2} d y .
$$

The right member can be made arbitrarily small by chosing first $\tau$ and then $\epsilon$. Thus relation (11.9) is proved for functions $u$ in $(5$.

A function $u$ in $\mathfrak{S}$ can be approximated by functions $\dot{u}$ in $\mathfrak{S}$ such that

$$
\left\|u-u^{\prime}\right\| \rightarrow 0 \text {. }
$$

Using relation (11.3) we may derive the inequality

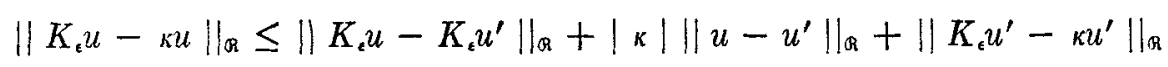

$$
\begin{aligned}
& \leq\left(k_{0}+|\kappa|\right)\left\|u-u^{\prime}\right\|_{\beta}+\left\|K_{\mathrm{s}} u^{\prime}-\kappa u^{\prime}\right\|_{\beta} \text {. }
\end{aligned}
$$

The last member can be made arbitrarily small by choosing first $u$ and then $\epsilon$. Thus relation (11.9) is proved to hold for the function $u$ in $\mathfrak{5}$.

\section{Mollifiers}

We now introduce a special set of integral operators, the "mollifiers." Their effect is to smooth out the functions on which they are applied; at the same time they approximate the identity.

Let $j(z)$ be a function of the number $z$ which possesses derivatives of every order and which satisfies the conditions

$$
\begin{gathered}
j(z) \geq 0, \\
\int_{-1}^{1} j(z) d z=1 .
\end{gathered}
$$

For any $\epsilon>0$ we then set

$$
j \cdot(y-\bar{y})=\epsilon^{-m} j\left(\epsilon^{-1}\left(y^{1}-\bar{y}^{1}+2 \epsilon\right)\right) \cdots j\left(\epsilon^{-1}\left(y^{m}-\bar{y}^{m}+2 \epsilon\right)\right)
$$


and define the mollifier $J_{\epsilon}$ as the integral operator with the kernel $j_{\epsilon}(y-\bar{y})$. This operator transforms a function $u$ in 5 into the function

$$
J_{\epsilon} u(y)=\int_{\mathbb{Q}} j_{\epsilon}(y-\bar{y}) u(\bar{y}) d \bar{y},
$$

which possesses continuous derivatives.

The terms $2 \epsilon$ were added in the arguments of the functions $j$ in expression (12.4) in order to make sure that $j_{\mathrm{c}}(y-\bar{y})=0$ when the point $\bar{y}$ is near the surface $\delta$. In the addition of these terms the present definition of the mollifiers differs from that given earlier [12B].

The operators $K_{\epsilon}=J_{\epsilon}$ and $k_{\epsilon}=J_{\epsilon}^{*}$ evidently enjoy property P I with

$$
k_{0}=\left\|J_{\epsilon}\right\|=\int\left|j_{\epsilon}(y-\bar{y})\right| d \bar{y}=\int j_{\epsilon}(y-\bar{y}) d \bar{y}=1 .
$$

Relation (11.3) takes the form

$$
\left\|J_{\epsilon} u\right\|_{G} \leq\|u\|_{G}, \quad\left\|J_{\epsilon}^{*} u\right\|_{G} \leq\|u\|_{G} .
$$

In order to establish further properties of the operator $J_{\epsilon}$ we make the following observations which involve the domains $R$ and $\Omega_{\tau}$ defined by (1.1) and $(1.1)_{\tau}$.

When the point $y$ is on the surface $s$, the set of points $y$ with $y^{r}<\bar{y}^{r}$, $r=1, \cdots, m$, lies outside of $R$. Hence we have

$$
j_{\epsilon}(y-\bar{y})=0 \quad \text { for } \quad \bar{y} \text { on } s, \quad y \text { in } R \text {; }
$$

of course, at the same time the derivatives of $j_{t}$ vanish; i.e.,

$$
\frac{\partial}{\partial \bar{y}^{r}} j_{\mathrm{t}}(y-\bar{y})=0 \quad \text { for } \quad \bar{y} \text { on } \mathrm{s}, \quad y \text { in } \mathrm{R} \text {. }
$$

When $y$ is on $J$, the set of points $\bar{y}$ with $\bar{y}^{r}>y^{r}, r=1, \cdots, m$ lies outside a. Hence we have

$$
j_{6}(y-\bar{y})=0 \quad \text { for } \quad y \text { on } J, \quad \bar{y} \text { in } R \text {. }
$$

When $\bar{y}$ is on $\Im$, the set of points $y$ with $\bar{y}^{r}-3 \epsilon<y^{r}<\bar{y}^{r}-\epsilon, r=1, \cdots, m$ is outside $\mathbb{R}_{3}$. Hence we have

$$
j_{e}(y-\bar{y})=0 \quad \text { for } \quad \bar{y} \text { on } \bar{J}, \quad y \text { in } \mathfrak{R}_{3 \mathrm{\varepsilon}} .
$$

When $y$ is in $R_{4 \varepsilon}$, the set of points $\bar{y}$ with $y^{r}+\epsilon<\bar{y}^{r}<\bar{y}^{r}+3 \epsilon$, $r=1, \cdots, m$, is in $R$. Hence we have

$$
\int j_{e}(y-\bar{y}) d \bar{y}=1 \quad \text { for } \quad y \text { in } \mathbb{R}_{4 e} .
$$


The statements (12.7) and (12.8) insure that the functions $J_{\varepsilon} u(y)$ vanish on the surface 3 and that the functions $J^{*} u(y)$ vanish on $s$;

$$
\begin{aligned}
& T J_{t}=0, \\
& S J_{t}^{*}=0 .
\end{aligned}
$$

In addition, it is clear that the functions $J_{\epsilon} u(y)$ and $J_{\epsilon}^{*} u(y)$ vanish also in the neighborhoods of $J$ and $S$ respectively.

Statement (12.8) implies the relation

$$
T J^{*} w=0
$$

for any function $w$ in $\mathbb{C}$ which vanishes in $R-\mathbb{R}_{3 \varepsilon}$; the function $T J_{\epsilon}^{*} w$ then vanishes even in a neighborhood of the surface $J$.

The fourth statement, (12.9) establishes property P II with $\tau(\epsilon)=4 \epsilon$, $\kappa=1$.

Property P III follows from the definition of $j_{\mathrm{e}}$.

We note that relation (11.9) takes the form

$$
\left\|J_{\varepsilon} u-u\right\|_{Q} \rightarrow 0 \quad \text { as } \quad \epsilon \rightarrow 0
$$

for all functions $u$ in $\mathfrak{S}$.

\section{Commutator Identity}

An important property of the operator $J_{\epsilon}$ is that it approximately commutes with the operator $E$. We shall show that under certain conditions the commutator $E J_{\varepsilon}-J_{\epsilon} E$ is equal to an integral operator to which the statements of Section 11 can be applied.

Evidently, the operator $E J_{\mathrm{s}}$ is an integral operator with the kernel

$$
\left[A^{\rho}(y) D_{\rho}+B(y)\right] j \cdot(y-\bar{y}) \text {. }
$$

Using the abbreviation $\bar{D}_{r}=\partial / \partial \bar{y}^{r}$ and using the relation

$$
\left(D_{r}+\bar{D}_{r}\right) j_{\cdot}(y-\bar{y})=0,
$$

we can write this kernel in the form

$$
\left[\bar{D}_{\rho} A^{\rho}(y)+B(y)\right] j_{\epsilon}(y-\bar{y}) .
$$

We express this fact symbolically by writing

$$
E J_{\mathrm{c}}=\left[\bar{D}_{p} A^{p}+B\right] J_{\epsilon} \cdot
$$

Similarly, the operator $E^{*} J_{\epsilon}^{*}$ is an integral operator with the kernel

$$
\left[-D_{\rho} A^{\rho}+B^{*}\right] j_{\epsilon}(\bar{y}-y) \text {. }
$$

The adjoint $\left(E^{*} J^{*}\right)^{*}$ of this integral operator has the kernel

$$
\left[-\bar{D}_{p} A^{p}(y)+B(\bar{y})\right] j_{\varepsilon}(y-\bar{y})
$$


therefore we write symbolically

$$
\left(E^{*} J_{\mathrm{t}}^{*}\right)^{*}=\left[-\bar{D}_{\rho} \bar{A}^{\rho}+\bar{B}\right] J_{e} \cdot .
$$

Combining (13.2) with (13.1) we obtain the identity

$$
E J_{\epsilon}-\left(E^{*} J_{\mathrm{\epsilon}}^{*}\right)^{*}=\left[\bar{D}_{\rho}\left(\bar{A}^{\rho}-A^{\rho}\right)-(\bar{B}-B) J_{\epsilon} \cdot .\right.
$$

Now, under certain circumstances the operator $\left(E^{*} J^{*}\right)^{*}$ equals the operator $J_{6} E$. Specifically we formulate

LEMma 13: The relation

$$
J_{\varepsilon} E u=\left(E^{*} J^{*}\right)^{*} u
$$

holds

(1) in $R$ for all functions $u$ in the space $B_{5}$ with $T u=0$,

(2) in $\mathfrak{B}_{3 \varepsilon}$ for all functions $u$ in $\mathbb{G}_{\mathfrak{\jmath}}$.

The qualifications "in $R^{\prime}$ " and "in $\Re_{3 \epsilon}$ " refer to the argument $y$ of the functions which result on both sides of (13.4).

In order to prove the lemma we first note that with arbitrary functions $w$

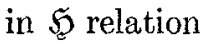

$$
\left(w, J_{\epsilon} E u\right)_{\Re}=\left(J^{*} w, E u\right)_{\Re},
$$

holds, as seen from (11.5). We next assume $w$ to be in $\mathfrak{C}_{1}$ with $S w=0$. Then we make use of the adjointness formula (3.6), which holds for $u$ in $\mathfrak{C}_{\jmath}$ since $S w=0$; it gives

$$
\left(w, J_{\epsilon} E u\right)_{\mathscr{Q}}=\left(E^{*} J_{\epsilon}^{*} w, u\right)_{\Re}+\left(J_{\varepsilon}^{*} w, A^{\mathrm{\rho}} \tau_{\rho} E u\right)_{\Im} .
$$

If $T u=0$, the last term on the right hand side vanishes. Otherwise, the last term vanishes because of relation (12.13). We restrict $w$ to vanish in $\mathbb{R}_{-}-\mathfrak{A}_{3 \epsilon}$; again using (11.5), we then obtain the formula

$$
\left(w, J_{\epsilon} E u\right)_{\Re}=\left(E^{*} J_{e}^{*} w, u\right)_{\Omega}=\left(w,\left(E^{*} J_{\epsilon}^{*}\right)^{*} u\right)_{\Omega} .
$$

If $w$ is arbitrary in $\mathfrak{S}_{1}$ with $S w=0$ we may apply Lemma 4 and conclude that relation (13.4) holds. If the function $w$ is arbitrary and lies in the domain $\mathcal{R}_{3 e}$, we may only conclude that this relation holds in $\mathbb{R}_{3 e}$. In any case, Lemma 13 is proved.

Combining it with identity (13.3) we have

THEOREM 13: The relation

$$
\left(E J_{\epsilon}-J_{\epsilon} E\right) u=\left[\bar{D}_{\rho}\left(\bar{A}^{\rho}-A^{\rho}\right)-(\bar{B}-B)\right] J_{\epsilon} \cdot
$$

\section{holds}

(1) in $\mathbb{R}$ for all functions $u$ in BS $_{\Im}$ with $T u=0$,

(2) in $\mathbb{R}_{3 \epsilon}$ for all functions in $\mathbb{G}_{\mathfrak{J}}$. 


\section{Conclusion}

One observes that relation (13.6) would reduce to relation $J_{\epsilon} E-E J_{\epsilon}=0$ if the matrices $A^{\rho}$ and $B$ were constant. In any case, though, the right member of this relation becomes small if $\epsilon$ is small.

The precise formulation of this statement will result from

Lemma 14: For a function $u$ in bj $_{3}$ relation

$$
\left\|\left[\bar{D}_{\rho}\left(\bar{A}^{\rho}-A^{\rho}\right)-(\bar{B}-B)\right] J_{\epsilon} \cdot u\right\|_{Q R} \rightarrow 0 \quad \text { as } \quad \epsilon \rightarrow 0
$$

holds.

To prove this statement we first derive the relation

$$
\left\|(B-\bar{B}) J_{\epsilon} \cdot u\right\|_{a} \rightarrow 0 \quad \text { as } \quad \epsilon \rightarrow 0,
$$

which, in obvious notation, involves the second part of the right member of formula (13.6).

Relation (14.2) follows from the estimate

$$
\left\|(B-\bar{B}) J_{\epsilon} \cdot u\right\|_{\Theta} \leq \beta(\epsilon)\left\|J_{\varepsilon} u\right\|_{\beta} \leq \beta(\epsilon)\|u\|_{\Omega},
$$

in which

$$
\beta(\epsilon)=\max |B(y)-B(\bar{y})| \quad \text { for } \quad\left|y^{r}-\bar{y}^{r}\right| \leq 3 \epsilon, \quad r=0, \cdots, m
$$

has been used. Clearly $\beta(\epsilon) \rightarrow 0$ as $\epsilon \rightarrow 0$ by virtue of the uniform continuity of $B(y)$ and (12.6).

Secondly, we shall prove that for any function $u$ in $\mathcal{O}_{5}$ the relation

$$
\left\|\bar{D}_{\rho}\left(A^{\rho}-\bar{A}^{p}\right) J_{\epsilon} \cdot u\right\|_{\Theta} \rightarrow 0 \quad \text { as } \quad \epsilon \rightarrow 0
$$

holds.

To this end we observe that the kernel

$$
k_{\epsilon}(y, \bar{y})=\bar{D}_{\rho}\left[A^{p}(y)-A^{p}(\bar{y})\right] j_{\epsilon}(y-\bar{y})
$$

of the operator $\bar{D}_{\rho}\left[A^{\circ}-\bar{A}^{\rho}\right] J_{\epsilon} \cdot$ enjoys properties P I, P II, P III of Section 11 with $\kappa=0$ and $\epsilon(\tau)=\tau / 4$.

It is obvious that $k_{t}$ has property P III.

In order to establish property P II with $\kappa=0, \epsilon(\tau)=\tau / 4$, we write $k_{\epsilon}(y, \bar{y})$ in the form

with

$$
k_{e}(y, \bar{y})=\bar{D}_{\rho} \Phi^{\rho}(y, \bar{y})
$$

$$
\Phi^{r}(y, \bar{y})=\left[A^{r}(y)-A^{r}(\bar{y})\right] j_{6}(y-\bar{y}),
$$

and observe

$$
\int k_{\epsilon}(y, \bar{y}) d \bar{y}=\int_{\mathcal{J}} \Phi^{\rho}(y, \bar{y}) d \bar{y}_{\rho}^{*}-\int_{\delta} \Phi^{\rho}(y, \bar{y}) d \bar{y}_{\rho}^{*} .
$$


Both terms on the right side vanish. For, $\Phi^{r}(y, \bar{y})=0$ for $\bar{y}$ on $\&$ by $(12.7)^{\prime}$ and, by (12.9), also for $\bar{y}$ on $J$ if $y$ lies in $\Re_{\tau}$ with $\tau \geq 3 \epsilon$.

In order to prove that the kernel $k_{*}(y, \bar{y})$ possesses property $\mathrm{P}$ I we split it up:

$$
k_{\epsilon}(y, \bar{y})=-\bar{D}_{\rho} A^{\rho}(\bar{y}) \cdot j_{\epsilon}(y-\bar{y})+\left[\left(A^{\rho}(y)-A^{\rho}(\bar{y})\right] \bar{D}_{\rho} j_{\epsilon}(y-\bar{y}) .\right.
$$

Using (12.6) we then obtain the estimate.

$$
\int_{\Omega}\left|\bar{D}_{\rho} A^{\rho}(\bar{y}) \cdot j_{\epsilon}(y-\bar{y})\right| d \bar{y} \leq\left|D_{\rho} A^{\rho} \cdot\right|_{\Theta} .
$$

Introducing the quantities

$\alpha(\epsilon)=\max \epsilon^{-1}\left|A^{k}(y)-A^{k}(\bar{y})\right| \quad$ for $\quad\left|y^{r}-\bar{y}^{r}\right| \leq 3 \epsilon, \quad r, k=1, \cdots, m$ and

$$
\eta(\epsilon)=\max \epsilon \int_{\Omega}\left|\bar{D}_{k} j_{\epsilon}(y-\bar{y})\right| d \bar{y} \quad \text { for } \quad y \quad \text { in } \quad \mathcal{R}, \quad k=1, \cdots, m
$$

we obtain the estimate

$$
\int\left[A^{\rho}(y)-A^{\rho}(\bar{y})\right] \bar{D}_{\rho} j_{\epsilon}(y-\bar{y}) \mid d \bar{y} \leq m \alpha(\epsilon) \eta(\epsilon) .
$$

The quantity $\alpha(\epsilon)$ is bounded because of the continuous differentiability of $A^{k}(y)$,

$$
\alpha(\epsilon) \leq 3 \max _{k, r}\left|D_{r} A^{k}\right|_{a r} .
$$

The quantity $\eta(\epsilon)$ is bounded because of

$$
\eta(\epsilon) \leq\left[\int_{-1}^{1}\left|\frac{d}{d z} j(z)\right| d z\right]^{m}=\eta_{0} .
$$

Inserting these estimates in (14.10) and combining them with (14.9) we obtain

$$
\int\left|k_{\epsilon}(y, \bar{y})\right| d \bar{y} \leq m\left[1+3 \eta_{0}\right] \max _{k, r}\left|D_{r} A^{k} \cdot\right| ;
$$

thus it is seen that the kernel $k_{t}(y, \bar{y})$ defined by (14.5) possesses property $\mathrm{P}$ I.

Having established the three properties of the operator $D_{\rho}\left[A^{\rho}-\vec{A}^{\rho}\right] J_{\epsilon} \cdot$ with the kernel $k_{\epsilon}(y, \bar{y})$, we know that relation (11.9) holds. Thus relation (14.4) follows. Combining it with relation (14.2), the statement of Lemma 14 results.

Combining the statement of Lemma 14 with that of Theorem 13 we obtain

TheOREM 14: For a function $u$ in $\mathbb{G}_{3}$ the relation

$$
\left\|E J_{\epsilon} u-J_{\epsilon} E u\right\|_{\Re} \rightarrow 0 \quad \text { as } \quad \epsilon \rightarrow 0
$$

holds if $T u=0$. Otherwise, the relation

$$
\left\|E J_{\iota} u-J_{\epsilon} E u\right\|_{B_{+}} \rightarrow 0 \quad \text { as } \quad \epsilon \rightarrow 0
$$

holds for any $\tau>0$. 
The statement of the Main Lemma 10, which refers to a function $u$ in $\mathfrak{B S}_{3}$ with $E u=0$ and $T u=0$, results from the first part of this theorem combined with relation (12.14) by setting

$$
u_{\bullet}=J_{\iota} u .
$$

For, if $u_{\mathrm{t}}$ is so chosen, we have $T u_{\mathrm{t}}=T J_{\mathrm{e}} u=0$ by (12.11) and relation (14.9) goes over into (10.2).

Thus the theorems and lemmas formulated in Sections 6, 8, 9 are established.

The second part of Theorem 14, involving relation $(14.9)_{r}$, will be used in Part II.

\section{PART II. DIFFERENTIABILITY}

\section{Differentiability Problem}

In this part we shall prove that the solutions $u$ of the equation $E u=f$ have derivatives if the right member $f$ and the initial values have derivatives. To have derivatives here means to admit the operators $D_{1}, \cdots, D_{m}$ in the strong sense introduced in Part I. The derivatives will further be proved to satisfy the differential equation which results by formal differentiation from $E u=f$. That the resulting differential equation possesses a solution follows from the existence theorem proved in Part I. We shall show that this solution consists of precisely the derivatives of the function $u$.

If we were to follow the procedure which is customary for ordinary differential equations, we would start with setting up a differential equation for the difference quotients of $u$. We do not find it feasible to proceed in this manner. Instead, we shall first approximate the solution $u$ of $E u=f$ by solutions $u^{\prime}$ of a finite difference equation $E^{\prime} u^{\prime}=f^{\prime}$. It is easy to set up an appropriate difference equation, pass to the limit, and prove that the limit function $u_{0}$ satisfies the equation $E u_{0}=f$ in the weak sense. The identity of the solutions $u_{0}$ and $u$ then follows from the theorem of the identity of the strong and weak extensions proved in Part I.

The finite difference equations are not easily adjusted to the initial surface S. Awkward special constructions would seem to be necessary unless the initial values of the functions $u^{\prime}$ and $f^{\prime}$ on $\delta$ were zero. With the aid of the solutions of the difference equations with initial values zero one can easily prove a restricted differentiability theorem which concerns solutions $u$ of $E u=0$ with $S u=0$. Fortunately, it is possible to reduce the general to the restricted differentiability theorem.

We mention incidentally that the method of finite differences used here to prove the differentiability theorem could also be used to design an existence proof independently of the method used in Part I.

To this end one would approximate the data which satisfy the conditions imposed in Part I by data which satisfy the conditions imposed in Part II. The 
solution constructed by the method of finite differences can be seen to have strong derivatives. Using the basic energy inequality of Part I one can construct a solution corresponding to the data imposed in Part I.

Since the identity of the strong and the weak extension can be derived from the existence theorem, the differentiability theorem could then be proved in the same way as is done below.

It may be said that approximation by solutions of difference equations would take the place of approximation by mollifiers in this different approach.

\section{The Space $\mathfrak{F}(D)$}

The space $\mathfrak{F}$ of functions which admit the operator $A D$ will from now on be denoted by $\mathfrak{F}(A D)$. We recall that every operator $E=A D+B$ is defined in $\mathfrak{F}(A D)$ whatever the continuous matrix $B$ may be. This remark applies in particular to the operator $-E^{*}=A D+D_{\rho} A^{\rho} \cdot-B^{*}$.

We recall that for $l=1, \cdots, m$ every operator $D_{l}$ is of the type $(E)$. The corresponding matrix $A^{r}$ is simply $A^{r}=1$ if $r=l, A^{r}=0$ if $r \neq l$, while $B=0$; the conditions imposed on $A^{r}$ and $B$ in Section 2 are of course satisfied. For the operator $D_{l}$ the adjointness identity (3.6) of Section 3 becomes

$$
\left(w, D_{\imath} u\right)_{\Re}+\left(D_{l} w, u\right)_{\Re}+\left(w, \sigma_{l} u\right)_{s}-\left(w, \tau_{l} u\right)_{\tau}=0 .
$$

Here $\sigma_{l}$ and $\tau_{l}$ are the numbers introduced in Section 3 .

The space $\mathfrak{F}$ with reference to the operator $D_{l}$ is denoted by $\mathfrak{F}\left(D_{l}\right)$; the intersection of the spaces $\mathfrak{F}\left(D_{1}\right), \cdots, \mathfrak{F}\left(D_{m}\right)$ will be denoted by $\mathfrak{F}(D)$. By $\mathfrak{F}(A D D)$ we denote the space of functions $u$ in $\mathfrak{F}(D)$ for which each $D_{l} u$ is in $\mathfrak{F}(A D)$.

By definition, every function $u$ in $\mathfrak{F}(D)$ can be approximated with respect to the norm \|\|$_{\mathscr{Q}}$ by functions in $\mathcal{E}_{1}$ together with each derivative $D_{l} u$. We shall show that for functions $u$ in $\mathfrak{F}(A D D)$ such an approximation can be performed simultaneously for $l=1, \cdots, m$, at least in subregions $\mathbb{R}_{r}$.

As in Section 1, we denote by $\mathbb{R}_{r}^{\prime}$ the domain which consists of all points $y$ that satisfy the $m$ conditions below:

$$
s^{r}\left(y_{r}^{*}\right)<y^{\tau}<t^{r}\left(y_{r}^{*}\right)-\tau, \quad r=1, \cdots, m .
$$

By $R_{r}$ we denote the closure of $R_{r}^{\prime}$.

We now proceed to derive

Lemma $16_{5}$ : Let $u$ be a function in $F\left(A^{\prime} D D\right)$ so that for $l=1, \cdots, m$ the function $D_{l} u$ is in a space $\mathfrak{F}\left(A^{\prime} D\right)$, where $A^{\prime}$ refers to any set of matrices of the type $(A)$. Then there exists a sequence of functions $u_{t}$ in $\mathfrak{G}_{1}$ with $D_{2} u$ in $\mathfrak{C}_{1}$ for $l=1, \cdots, m$, such that for $\tau>0$

$$
\begin{gathered}
\left\|u_{\mathrm{e}}-u\right\|_{Q_{r}} \rightarrow 0, \\
\left\|D_{l} u_{\epsilon}-D_{l} u\right\|_{Q_{r}} \rightarrow 0,
\end{gathered}
$$




$$
\left\|E D_{l} u_{\mathrm{s}}-E D_{t} u\right\|_{Q_{\mathrm{r}}} \rightarrow 0
$$

$$
\begin{gathered}
\left\|u_{\epsilon}-u\right\|_{\delta_{r}} \rightarrow 0, \\
\left\|D_{l} u_{\epsilon}-D_{l} u\right\|_{\delta_{r}} \rightarrow 0 \quad \text { as } \quad \epsilon \rightarrow 0 .
\end{gathered}
$$

If $T u=0, T D_{l} u=0$, the functions $u_{\mathrm{\epsilon}}$ vanish in a neighborhood of $J$ and the relations (16.3) hold for $\tau=0$, i.e. for $R$ instead of $R_{\tau}$.

To prove Lemma $16_{3}$ we use the mollifiers $J_{\epsilon}$ (see Section 12) and state that the functions $u_{\varepsilon}=J_{\epsilon} u$ possess the desired properties. Indeed, $J_{\ell} u$ vanishes in a neighborhood of $J$, as stated in the remark following formula (12.12). Relation (14.6) ${ }_{1}$ follows immediately from (12.14). In case $T u=0$, relation (13.6), applied to $D_{l}$ instead of $E$, reduces to

$$
D_{\imath} J_{\epsilon} u=J_{\varepsilon} D_{\imath} u \text {. }
$$

Without assuming $T u=0$, this relation was only proved to hold in a subdomain $\mathbb{R}_{3 \mathrm{e}}$.

Relation (14.9) $)_{3 e}$, applied to $D_{\imath} u$ instead of $u$, yields the relation

$$
\left\|E J_{\epsilon} D_{l} u-J_{\epsilon} E D_{\imath} u\right\|_{\mathcal{B}_{\imath \ell}} \rightarrow 0 \quad \text { as } \epsilon \rightarrow 0
$$

and hence, by $(16.4)_{1}$,

$$
\left\|E D_{l} J_{\epsilon} u-J_{t} E D_{l} u\right\|_{\AA_{2}} \rightarrow 0 \quad \text { as } \quad \epsilon \rightarrow 0 ;
$$

if $T u=T D_{l} u=0$, relation $(16.4)_{2}$ holds for $R$ in place of $\Omega_{3 \epsilon}$.

From $(16.4)_{1},(16.4)_{2}$ and $(12.14)$ the relations $(16.3)_{2}$ and $(16.3)_{3}$ follow. Relations (16.3) $)_{4}$ and $(16.3)_{5}$ for $\tau>0$ can be derived from the latter two relations with the aid of inequality $(7.1)_{\jmath}$ if $T u=T D_{\imath} u=0$. Otherwise we use the inequality

$$
\|u\|_{\delta_{\tau}}^{2} \leq \gamma\|E u\|_{Q}^{2}+\Omega \gamma\|u\|_{\AA}^{2},
$$

which follows from inequality (7.8) when $\omega$ is chosen as $\omega=1$ in $\delta_{r}$. Inequality (16.5) applied to $\mathscr{R}_{\tau^{\prime}}$ with $0<\tau^{\prime}<\tau$, instead of $R$, to $u$ and $D_{l} u$ or $D_{l} u$ and $E D_{l} u$ instead of $u$ and $E u$, yields $(16.3)_{4}$ and $(16.3)_{5}$. Thus Lemma 16 is proved.

It must be remarked that the initial value $S u$ of a function $u$ in $\mathfrak{F}(D)$ depends on $l$, since $S u$ is assigned to $u$ with reference to each operator $D_{l} u$ in place of $E$. For the continuously differentiable function $u_{\epsilon}$, however, $S u_{\epsilon}$ is independent of $l$; hence relation $(16.3)_{4}$ in Lemma $16_{3}$ implies that $S u$ is independent of $l$. The same is true for $S D_{r} u$ in view of $(16.3)_{5}$.

Note also that the space $\mathfrak{F}(D)$ is contained in $\mathfrak{F}(A D)$; therefore $A D=A^{\rho} D_{\rho}$ and hence $E u=A^{\rho} D_{\rho} u+B u$ for $u$ in $\mathfrak{F}(D)$. Indeed, the quantity $f=A^{\rho} D_{\rho} u$ with $\tilde{g}=S u$ evidently satisfies relation $(9.1)_{s}$ for every $w$ in $\mathbb{S}_{1}$ with $T w=0$; hence $u$ is in $\mathfrak{G}_{s}$ and, according to Theorem $9_{s}^{I}, u$ is in $\mathfrak{F}(A D)$. 


\section{Differentiability Theorem}

The main theorem of Part II to be formulated in this section states under what circumstances the solution $u$ of the initial problem $E u=f, S u=S g$, formulated in Section 6, possesses strong derivatives, i.e. lies in the space $\mathfrak{F}(D)$. We shall make four assumptions: The right member $f$ is itself in $\mathfrak{F}(D)$. The initial value of $u$ is at the same time the initial value of a function $g$ which itself is in $\mathfrak{F}(D)$ and whose derivatives have initial values in $\mathfrak{W}_{s}$. The matrix $B$ possesses continuous derivatives. The initial surface $\mathcal{S}$ is sufficiently smooth. Specifically:

I $f$ is in $\mathfrak{F}(D)$.

II There is a function $g$ in $\mathfrak{F}(D)$ whose derivatives $D_{l} g$ are in a space $\mathfrak{F}\left(A^{\prime} D\right)$ such that $S u=S g$. Here $A^{\prime}$ refers to any set of matrices of the type $(A)$.

This form of condition II was chosen in order to make sure that the derivatives $D_{l} u$ possess initial values $S D_{l} g$. We shall express the initial values $S D_{r} u$ of $D_{r} u$ in terms of the initial values $S D_{l} g$.

It may appear unnatural to characterize the behavior of the function $u$ on $\delta$ by means of a function defined in $R$ instead of characterizing this behavior directly by differentiability conditions on $S$. The latter possibility may, however, be subsumed under our definition. A function defined on $S$ need only be continued as a function $g$ in $R$ so as to be independent of $y_{1}$; then $D_{1} g=0$. Our requirement that $g$ be in $\mathscr{F}(D)$ is then equivalent to certain differentiability conditions on $\delta$ and the additional requirement that $D_{l} g$ be in $\mathfrak{F}\left(D_{1}\right)$ is no restriction in view of $D_{1} D_{l} g=0$.

III The matrices $B(y)$ are in $\mathfrak{G}_{1}$.

IV There exists a function $\sigma(y)$ in $\mathfrak{S}_{3}$ which vanishes on $\mathbb{S}$,

$$
\sigma(y)=0 \text { on } s,
$$

and whose derivatives

$$
\sigma_{r}(y)=D_{r} \sigma(y)
$$

are positive on $S$,

$$
\sigma_{r}(y)>0 \quad \text { on } s, \quad r=1, \cdots, m .
$$

Evidently, the numbers $\sigma_{r}$ introduced by (17.2) may be taken as the numbers $\sigma_{r}$ introduced in Section 3.

The condition that the function $\sigma(y)$ be in $\mathfrak{C}_{3}$ is made for convenience; it is stronger than necessary. It could be shown-but we shall not do so-that it would be sufficient to assume that $\sigma(y)$ is in $\mathscr{S}_{1}$.

The main theorem of Part II will now be formulated as

Differentiability Theorem $17_{\mathrm{s}}$ : Assume that the initial surface $\mathbb{S}$ of the lens-shaped region $R$ satisfies condition $I V$ and that the operator $E=A D+B$ of type $(E)$ satisfies condition $I I I$. Suppose that $u$ is a function in $\mathfrak{F}(A D)$ for which 
the function $E u$ is in $F(D)$ and whose initial values Su satisfy condition $I I$. Then the function $u$ belongs to the space $\mathfrak{F}(D)$ and satisfies the equations

$$
E D_{l} u=D_{l} E u-D_{l} A^{p} \cdot D_{\rho} u-D_{l} B \cdot u
$$

and

$$
S D_{l} u=S\left\{D_{l} g+\left(A^{\rho} \sigma_{\beta}\right)^{-1} \sigma_{l}[E u-E g]\right\} .
$$

Here the quantities $\sigma_{l}$ are the numbers introduced by (17.2).

Relation (17.4) certainly holds for functions $u$ in $\mathfrak{S}_{2}$, as is immediately verified by formal differentiation. Relation (17.5) enables one to express the initial values of the derivatives $D_{l} u$ in terms of $E u$ and the initial values $g$ of $u$. Note that the expression $S\left\{D_{l} g-\left(A^{p} \sigma_{\rho}\right)^{-1} \sigma E g\right\}$ actually involves the derivatives of $g$ only in directions within the surface $s$. We have

$$
A^{\rho} \sigma_{\rho} D_{l} g-\sigma E g=A^{\rho}\left[\sigma_{\rho} D_{l}-\sigma_{l} D_{\rho}\right] g-\sigma_{l} B g
$$

and the expression $\sigma_{\rho} D_{l}-\sigma_{l} D_{\rho}$ represents differentiation in a direction in the surface $s$; cf. (3.7).

We have already indicated in Section 15 that, instead of proving Theorem 17 directly, we shall reduce this theorem to a weakened form in which the initial values of $u$ and $E u$ are zero. We formulate this weakened theorem separately:

Special Differentiabicity Theorem $17_{8}^{0}:$ Assume that the operator $E=A D+B$ of type $(E)$ satisfies condition III. Let $u$ be a function in $\mathfrak{F}(A D)$ such that $E u$ is in $\mathfrak{F}(D)$ and

$$
\begin{aligned}
u=0 & \text { on } \$ \\
E u & =0 \quad \text { on } \quad \text { s. }
\end{aligned}
$$

Then the function $u$ belongs to the space $\mathfrak{F}(D)$; further $D_{\imath} u$ belongs to $\mathfrak{F}(A D)$ and satisfies the equation (17.4) and moreover

$$
D_{l} u=0 \quad \text { on } \mathrm{s} \text {. }
$$

Note that in this special theorem condition IV is not imposed.

We shall first put the relations stated in these two theorems into a somewhat different form, then prove Theorem $17^{\circ}$, and finally reduce Theorem 17 to $17^{\circ}$.

\section{Operators $\hat{E}$ and $\hat{D}$}

It is convenient to introduce functions which are systems

$$
\hat{u}=\left[u, u_{l}\right]=\left[u_{0}, u_{1}, \cdots, u_{m}\right]
$$

of $m+1$ functions in any of the spaces considered. We also characterize the corresponding spaces of systems $\hat{u}$ by a "caret." E.g. if $u$ and $u_{l}, l=1, \cdots, m$, 
are in $\mathfrak{g}$ we say $\hat{u}$ is in $\hat{\mathfrak{F}}$. For systems $\hat{u}$ we introduce the matrices

$$
\hat{A}^{r}=\left(\begin{array}{cccc}
A^{r} & 0 & \cdots & 0 \\
0 & A^{r} & \cdots & 0 \\
\cdot & \cdot & \cdots & \cdot \\
0 & 0 & \cdots & A^{r}
\end{array}\right),
$$

$$
\hat{B}=\left(\begin{array}{cccc}
B & 0 & \cdots & 0 \\
D_{1} B . & D_{1} A^{1} \cdot+B & \ldots & D_{1} A^{m} \cdot \\
\cdot & \cdot & \ldots & \cdot \\
D_{m} B . & . & \ldots & D_{m} A^{m}+B
\end{array}\right) .
$$

The operator $\hat{E}=\hat{A} D+\hat{B}$ acting on functions $u$ can then be formed. Specifically, we have

$$
\hat{E} \hat{u}=\left[E u_{0}, E u_{l}+D_{l} A^{\rho} \cdot u_{p}+D_{l} B \cdot u_{0}\right] .
$$

The space of functions $\hat{u}$ for which the operator $\hat{E}$ is defined strongly will be called $\mathfrak{F}(\hat{A} D)$.

For functions $u$ in $\mathfrak{F}(D)$ we introduce the operator $\hat{D}$ by means of

$$
\hat{D} u=\left[u, D_{\imath} u\right]=\left[u, D_{1} u, \cdots, D_{m} u\right] .
$$

For functions $u$ in $\mathfrak{F}(D D)$ the identity

$$
\hat{E} \hat{D} u=\hat{D} E u
$$

holds. In fact, as seen from (18.3), equation (17.4) together with $\hat{E} u=E u$ is equivalent with this identity.

We introduce the vector

$$
\hat{\sigma}=\left\{0, \sigma_{1}, \cdots, \sigma_{m}\right\}
$$

so that we may write

$$
(\hat{v}, \hat{\sigma} u)_{\delta}=\left(v_{\rho}, \sigma_{\rho} u\right)_{s} .
$$

Using this vector we can express the initial conditions $S u=S g$ and (17.5) as

$$
S \hat{D} u=S\left\{\hat{D} g+\left(A^{\rho} \sigma_{\rho}\right)^{-1} \hat{\sigma}(E u-E g)\right\} .
$$

Let $u$ be the function with which Theorem $17_{\mathrm{s}}$ is concerned, and let $g$ be the function entering the formulation of this theorem, which agrees with $u$ on the surface $\delta$.

Using these functions we may formulate the problem of finding a function 
$\hat{u}$ in $\hat{\mathfrak{F}}(\hat{A} D)$ which satisfies the differential equation

$$
\hat{E} \hat{u}=\hat{D} E u
$$

and the initial conditions

$$
S \hat{u}=S\left\{\hat{D} g+\left(A^{\rho} \sigma_{\rho}\right)^{-1} \hat{\sigma}(E u-E g)\right\} .
$$

This problem is of the type for which in Part I the unique existence of a solution was proved, ef. Theorem $6_{s}^{E}$. Let the function $\hat{u}$ in $\hat{\mathfrak{F}}(\hat{A} D)$ be this solution. Then, evidently, the statement of Theorem 17 is simply equivalent with the statement that the function $u$ lies in the space $\mathfrak{F}(D)$ and that

$$
\hat{D} u=\hat{u} \text {. }
$$

A similar formulation could be given for Theorem $17_{s}^{0}$, but we shall not need it.

\section{Difference Operators}

In order to prove Theorem $17_{\mathrm{s}}^{0}$ we replace differential operators by difference operators in such a way that

(i) the existence of the solution to the corresponding initial value problem is obvious,

(ii) the properties corresponding to those stated in Theorem $17_{\S}^{0}$ are obvious for these solutions,

(iii) when the mesh width approaches zero, a subsequence of such solutions converges weakly to a limit function in $B(A D)$ possessing the desired properties. By virtue of the coincidence of the operators $E$ in the weak and strong sense, Theorem $17_{3}^{0}$ will follow.

We introduce the translation $[r]$ defined by

$$
\begin{gathered}
{[r] y^{r}=y^{r}-\lambda \quad \text { and } \quad[r] y^{l}=y^{l} \quad \text { if } \quad l \neq r,} \\
{[r]\left\{y^{1}, \cdots, y^{m}\right\}=\left\{[r] y^{1}, \cdots,[r] y^{m}\right\} .}
\end{gathered}
$$

Here the "mesh width" $\lambda$ is any positive number at our disposal. The translation $[r]^{-1}$ is defined, similarly, by substituting $-\lambda$ for $\lambda$.

From the assumptions about the nature of the region $R$, made in Section 1, one easily deduces the

REMARK: If the points $y$ and $[l][r] y$ lie in $\mathcal{A}$, then $[r] y$ and [l]y also lie in $R$; if the points $y$ and $[l]^{-1}[r]^{-1} y$ lie in $\Re$, then $[r]^{-1} y$ and $[l]^{-1} y$ also lie in $R$.

To prove this we need only observe: Suppose for two points $y$ and $\tilde{y}$ on $\delta$

$$
y^{\mu}=\tilde{y}^{\mu} \quad \text { for } \quad \mu \neq r \neq l \quad \text { and } \quad y^{l}<\tilde{y}^{l} ;
$$

then

$$
\sigma^{r}\left(y_{r}^{*}\right)>\sigma^{r}\left(y_{r}^{*}\right)
$$


The symbol $[r]$ is also used to denote the operation which transforms a function $u(y)$ in $\sqrt{ }$ into the function

$$
[r] u(y)=u([r] y)
$$

provided the point $[r] y$ lies in $R$. We define

$$
[r] u(y)=u([r] y)=0
$$

if $[r] y$ lies outside $R$. Similarly, for functions $v$ in $\mathbb{C}$ we define the operator $[r]^{-1}$ by

$$
[r]^{-1} v(y)=v\left([r]^{-1} y\right),
$$

provided $[r]^{-1} y$ lies in $\mathcal{R}$, and by

$$
[r]^{-1} v(y)=v\left([r]^{-1} y\right)=0
$$

if $[r]^{-1} y$ lies outside $R$.

We easily verify the identity

$$
\left([r]^{-1} v, u\right)_{Q}=(v,[r] u)_{Q}
$$

for any functions $u, v$, in $\mathfrak{C}$; it expresses the fact that the operator $[r]^{-1}$ is the adjoint of $[r]$. For any function in $\mathbb{E}$ we further have

$$
([r] u,[r] u)_{\circledast} \leq(u, u)_{\circledast}
$$

and, more generally,

$$
([r] u,[r] K u)_{a} \leq(u, K u)_{\curvearrowleft},
$$

whenever $K=K(y)$ is a continuous bounded non-negative symmetric matrix.

By virtue of inequality (19.6) we can extend the operator $[r]$ as a bounded operator to the whole Hilbert space $\$$; the same is, of course, true of the operator

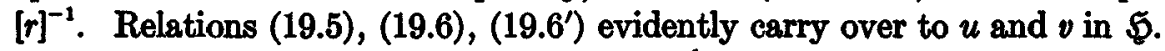

With the aid of the translations $[r],[r]^{-1}$ we define the difference operators

$$
\begin{aligned}
& \Delta_{r}=\lambda^{-1}(1-[r]), \\
& \Delta_{r}^{*}=\lambda^{-1}\left(1-[r]^{-1}\right) ;
\end{aligned}
$$

they serve as substitutes for the differential operators $D_{r}$ and $-D_{r}$.

Let $f$ be a function in $\mathfrak{C}_{1}$ which vanishes on $J$. Since $f$ is continuous across I when we set $f([r] y)=0$ in agreement with (19.4), it is clear that $\Delta_{r} f(y)$ can be written as

$$
\Delta_{r} f(y)=\lambda^{-1} \int_{0}^{\lambda} D_{r} f\left(y_{r}^{*}, y^{*}-\mu\right) d \mu,
$$

for every point $y$ in $R$. As a consequence, the inequality

$$
\left\|\Delta_{r} f\right\|_{Q} \leq\left\|D_{r} f\right\|_{a}
$$


and the relation

$$
\left\|\Delta_{r} f-D_{r} f\right\|_{a} \rightarrow 0 \quad \text { as } \quad \lambda \rightarrow 0
$$

hold. By virtue of the definition of the strong extension of the operator $D$, inequality (19.10), and hence also relation (19.9), carry over to functions $f$ in $\mathfrak{F}\left(D_{\text {r }}\right)$ with $T f=0$.

Let $A^{r}=A^{r}(y), r=1, \cdots, m$, be non-negative symmetric matrices with continuous derivatives defined in $G$ such that with an appropriate positive constant $a$ the matrix $\sum_{p} A^{p}-a$ is non-negative, cf. (2.3).

Furthermore, let $B_{\lambda}^{r}=B_{\lambda}^{r}(y)$ be a set of continuous matrices defined in $q$. We assume that there is a constant $b$ such that

$$
\left|B_{\lambda}^{r}(y)\right| \leq b, \quad r=1, \cdots, m, \quad \text { for } \quad y \text { in } a .
$$

Then we introduce the difference operator $E_{\lambda}$ as the expression

$$
E_{\lambda}=A^{\rho} \Delta_{\rho}+B_{\lambda}^{\rho}[\rho],
$$

in which summation with respect to $\rho$ is implied in both terms. This operator transforms the function $u(y)$ into the function

$$
E_{\lambda} u(y)=\lambda^{-1} A^{\rho}(y) u(y)-\lambda^{-1} A^{\rho}(y) u([\rho] y)+B_{\lambda}^{\rho}(y) u([\rho] y) .
$$

Let $f_{r}(y)$ be a function in (5. Then we ask for a function $u(y)=u_{r}(y)$ in $\mathbb{R}$ which satisfies the equation

$$
E_{\lambda} u=f_{\lambda} .
$$

Note that the stipulation (19.4) takes the place of an initial condition on $\mathrm{S}$.

The expression $E_{\mu} u(y)$ involves the values of $u$ at the points $y,[1] y, \cdots$, [m]y. The coefficient of $u(y)$ is the matrix $\lambda^{-1} \sum_{\rho} A^{\rho}(y)$; because of (2.3) it possesses an inverse. Therefore, since the value of $E_{\lambda} u=f_{\lambda}$ is given at each point $y$, the value of $u$ at the point $y$ can be expressed through the values of $u$ at the $m$ point [r]y. Successive application of this reduction ends in a finite number of steps because of condition (19.4). Thus one can express the value of $u$ at any point of $R$ in terms of the values of $f_{\lambda}$ in $R$. In this way the solution of the initial value problem described by (19.15), (19.14), is constructed. It is evident that this solution is continuous.

In the following we shall use the operator

$$
E_{\lambda}^{*}=\Delta_{\rho}^{*} A^{p}+[\rho]^{-1} B_{\lambda}^{\rho^{*}} ;
$$

it is the adjoint of the operator $E_{\lambda}$, as seen from the identity

$$
(E * v, u)_{a}=\left(v, E_{\lambda} u\right)_{\Re}
$$

which follows from identity (19.5).

We proceed to derive an inequality by which the norm $\|u\|_{a}$ of a function $u$ is estimated by the norm $\left\|E_{\lambda} u\right\|_{a}$ of $E_{\lambda} u$; it is analogous to inequality (7.1) for functions $u$ in $\mathfrak{F}$ with $S u=0$. 
In doing this we shall employ the function

$$
q(y)=\exp \left\{-\theta \sum_{\rho} y^{\rho}\right\},
$$

keeping the positive number $\theta$ at our disposal. We note that this function satisfies the relation

$$
[r] q(y)=\exp \{-\theta \lambda\} q(y) .
$$

We now write the relation $A^{\rho} \Delta_{\rho} u+B_{\lambda}^{\rho}[\rho] u=E_{\lambda} u$, cf. (19.12), in the form

$$
\sum_{\rho} A^{\rho} u=\sum_{\rho} A^{\rho}[\rho] u-\lambda \sum_{\rho} B_{\lambda}^{\rho}[\rho] u+\lambda E_{\lambda} u,
$$

multiply it by $q^{2} u$, and integrate over $\mathfrak{R}$. The resulting relation may be written in the form

$$
\sum_{\rho}\left(q u, A^{\rho} q u\right)_{\Re}=\left(q u, A^{\rho} q[\rho] u\right)_{\Re}-\lambda\left(q u, B_{\lambda}^{\rho} q[\rho] u\right)_{\Re}+\lambda\left(q u, q E_{\lambda} u\right)_{\Re}
$$

or, using formula (19.17), in the form

$$
\begin{aligned}
\sum_{\rho}\left(q u, A^{p} q u\right)_{\Theta}=\exp & \{-\theta \lambda\}\left(q u, A^{\rho}[\rho] q u\right)_{\Theta} \\
& -\lambda \exp \{-\theta \lambda\}\left(q u, B_{\lambda}^{\rho}[\rho] q u\right)_{\mathscr{Q}}+\lambda\left(q u, q E_{\lambda} u\right)_{\mathscr{G}} .
\end{aligned}
$$

At this place we make essential use of the assumption that the matrices $A^{r}(y)$ are non-negative definite: we infer from it that the estimates

$$
2\left(q u, A^{r}[r] q u\right)_{\circledast} \leq \exp \theta \lambda\left(q u, A^{r} q u\right)_{\mathscr{Q}}+\exp \{-\theta \lambda\}\left([r] q u, A^{r}[r] q u\right)_{\mathscr{Q}}
$$

hold for $r=1, \cdots, m$. Insertion of this inequality in (19.18) leads to the relation

$$
\sum_{\rho}\left(q u, A^{\rho} q u\right)_{\Re} \leq \exp \{-2 \theta \lambda\} \sum_{\rho}\left([\rho] q u, A^{\rho}[\rho] q u\right)_{\Re}
$$

We now use the relation

$$
-2 \lambda \exp \{-\theta \lambda\}\left(q u, B_{\lambda}^{p}[\rho] q u\right)_{\mathfrak{R}}+2 \lambda\left(q u, q E_{\lambda} u\right)_{\Re} .
$$

$$
A^{\tau}[r]=[r] A^{r}-\lambda \Delta_{r} A^{r} \cdot[r],
$$

which follows from (19.7) and write accordingly

$$
\left([r] q u, A^{r}[r] q u\right)_{\mathscr{A}}=\left([r] q u,[r] A^{r} q u\right)_{\Re}-\lambda\left([r] q u, \Delta_{r} A^{r} \cdot[r] q u\right)_{\mathscr{R}} .
$$

The value of the difference quotient $\Delta_{r} A^{r}$. at points $y$ for which $[r] y$ lies outside of $R$ is not defined; but the expression $\left([r] q u, \Delta_{r} A^{r} \cdot[r] q u\right)_{\mathscr{R}}$ is independent of these values since $[r] q u$ vanishes at such points.

Clearly, since $D_{r} A^{r}$. is assumed to be bounded, there is an upper bound for the modulus $\mid \Delta_{r} A^{r}$. $\mid$ of the matrix $\Delta_{r} A^{r}$. at all points $y$ in $R$ with $[r] y$ in $R$ independently of the mesh width $\lambda$; we denote it by $c$. Accordingly,

$$
\left|\Delta_{r} A^{r} \cdot\right| \leq c \quad \text { for } \quad y \text { in } R \quad \text { if }[r] y \text { in } R \text {. }
$$


Using this bound, thee last trm of (19.20) ean be estimated in the form

$$
\left|\left([r] q u, \Delta_{r} A^{r} \cdot[r] q u\right)_{\mathscr{Q}}\right| \leq c([r] q u,[r] q u)_{\mathscr{A}} .
$$

Using this estimate and the bound (19.10) we obtain from relations (19.19) and (19.20) in an obvious manner the estimate

$$
\begin{aligned}
\sum_{\rho}\left(q u, A^{\rho} q u\right)_{\mathscr{R}} \leq \exp \{ & -2 \theta \lambda\} \sum_{\rho}\left([\rho] q u,[\rho] A^{\rho} q u\right)_{\mathfrak{R}} \\
& +\lambda(c+b) \sum_{\rho}([\rho] q u,[\rho] q u)_{\Re} \\
& +\lambda(m b+1)(q u, q u)_{\mathscr{G}}+\lambda\left(q E_{\lambda} u, q E_{\lambda} u\right)_{\mathscr{R}} .
\end{aligned}
$$

We now employ inequality (19.6) with $k=q A^{r}$ and $k=q$; we then obtain from (19.21)

$$
\begin{aligned}
& {[1-\exp \{-2 \theta \lambda\}] \sum_{\rho}\left(q u, A^{p} q u\right)_{\mathbb{R}}} \\
& \quad \leq \lambda m(m c+2 m b+1)(q u, q u)_{\mathfrak{R}}+\lambda\left(q E_{\lambda} u, q E_{\lambda} u\right)_{\mathscr{R}} .
\end{aligned}
$$

whence, using (2.2), we find

$(19.22)\{[1-\exp \{-2 \theta \lambda\}] a-\lambda(m c+2 m b+1)\}(q u, q u)_{\mathbb{R}} \leq \lambda\left(q E_{\lambda} u, q E_{\lambda} u\right)_{\mathbb{R}}$.

We now choose

$$
\theta=2(m c+2 m b+1)
$$

and then require $\lambda$ to be so small that

$$
\exp \{2 \lambda \theta\} \leq 2
$$

so that

$$
1-\exp \{-2 \theta \lambda\} \geq \theta \lambda .
$$

From (19.22) we then have

$$
\theta(q u, q u)_{\propto} \leq 2\left(q E_{\lambda} u, q E_{\lambda} u\right)_{\leftrightarrow} .
$$

\section{Setting}

$$
\gamma^{2}=2 \theta^{-1} \max _{\propto} q / \min _{\sigma} q,
$$

we finally obtain the inequality

$$
(u, u)_{\Re} \leq \gamma^{2}\left(E_{\lambda} u, E_{\lambda} u\right)_{\Re}
$$

for any function $u$ in $\mathfrak{S}$.

For the solution $u=u_{\lambda}$ of equation (19.13) we thus have

$$
\left\|u_{\lambda}\right\|_{R} \leq \gamma\left\|f_{\lambda}\right\|_{\beta} \text {. }
$$


This solution was constructed under the assumption that the right member $f_{\lambda}$ belongs to $\mathfrak{E}$. Since every function $f_{\lambda}$ in $\mathfrak{S}$ can be approximated in the sense of the \|\|$_{\alpha}$-norm by functions in $\mathbb{C}$, we may state

THeOREM 19: To every function $f_{\lambda}$ in $\mathfrak{S}$ a solution $u=u_{\lambda}$ of the difference equation (19.23), $E_{\lambda} u=f_{\lambda}$, exists which satisfies condition (19.4). For this solution inequality (19.26) holds.

A general remark may be added concerning the derivation of inequality (19.26). In an earlier work on hyperbolic difference equations [5C] the difference operator was set up in such a way that in the analogue of identity (19.18) the first term on its right member involved a positive definite form. Such a set-up would also be possible in the present, more general, problem. To this end one need only replace the operator $\Delta_{p}$ in expression (19.11) by the average of the $2^{m-1}$ operators of the form $\Delta_{\rho}, \Delta_{\rho}\left[\mu_{1}\right], \cdots, \Delta_{\rho}\left[\mu_{1}\right]\left[\mu_{2}\right], \cdots$, in which $\left[\mu_{1}\right],\left[\mu_{2}\right],\left[\mu_{3}\right], \cdots$ represent distinct translations different from $[\rho]$. The resulting difference operator connects the value of $u$ at the point $y$ with the values of $u$ at all points of the cube spanned by the points $y,[1] y, \cdots[1] \cdots$ $[m] y$. The form of the operator (19.11) which we have chosen is certainly simpler and, although it does not lead to an identity of the type (7.2) involving positive definite forms associated with the surfaces $\delta$ and $J$, it is still sufficient to derive inequality (19.26).

\section{Limit Process}

We now investigate what happens when the mesh width $\lambda$ approaches zero. We say that functions $\phi_{0}(y)$ converge mod $s$ and $\xi$ to a continuous limit function $\phi_{0}(y)$ if they converge uniformly to $\phi_{0}(y)$ in $R$ outside every neighborhood of $\&$ and I respectively while $\left|\phi_{\Lambda}(y)\right|$ remains bounded in such neighborhoods.

In this sense the matrices $\Delta_{r} A^{r}$. converge mod $J$ to the matrices $D_{r} A^{r}$. since it was assumed that the $A^{r}$ possess continuous derivatives. As regards the matrices $B_{\lambda}^{r}$ we stipulate that they approach mod $\mathcal{I}$ continuous matrices $B^{r}$ for which

$$
\sum_{\rho} B^{\rho}=B .
$$

We further assume that the common bound $b$ of $\left|B_{\lambda}^{p}\right|$ is independent of $\lambda$; cf. (19.10). The independence of the constant $c$ of $\lambda$ has already been established, ef. (19.21).

Further we assume that the norm $\left\|f_{\lambda}\right\|_{\Theta}$ of the right member $f_{\lambda}$ of equation (19.13) remains bounded,

$$
\left\|f_{\lambda}\right\|_{\Re} \leq k .
$$

By virtue of inequality (19.27) the norm of the solution $u=u_{\lambda}$ of the initial value problem (19.13), (19.4) is then also bounded,

$$
\left\|u_{\lambda}\right\|_{\Re}=\lambda k \text {. }
$$


A well-known consequence of inequalities (20.3) and (20.2) is that a sequence $\lambda \rightarrow 0$ exists such that $u_{\lambda}$ and $E_{\lambda} u_{\lambda}$ converge weakly; i.e. functions $u_{0}$ and $f_{0}$ in $\mathfrak{S}$ exist such that

$$
\begin{gathered}
\left(w_{\lambda}, u_{\lambda}\right)_{R} \rightarrow\left(w_{0}, u_{0}\right)_{R}, \\
\left(w_{\lambda}, E_{\lambda} u_{\lambda}\right)_{\Re} \rightarrow\left(w_{0}, f_{0}\right)_{\mathscr{R}}, \quad \text { as } \quad \lambda \rightarrow 0,
\end{gathered}
$$

whenever $w_{\lambda}$ and $w_{0}$ are functions in $\mathfrak{S}$ for which

$$
\left\|w_{\lambda}-w_{0}\right\|_{\text {A }} \rightarrow 0 \text { as } \quad \lambda \rightarrow 0 \text {. }
$$

Let $v$ be a function in $\mathcal{S}_{1}$ which vanishes on $\mathbb{S}$. Note that the operator $E_{\lambda}^{*}$ involves difference operators $\Delta_{r}^{*}$, which connect points across $S$ but not points across 3 . Therefore the function $E^{*} v$ converges uniformly in $\&$ to $E^{*} v$. Hence, when we set $w_{\lambda}=E_{\lambda}^{*} v$, we obtain from (20.14) the relation

$$
\left(E_{\lambda}^{*} v, u \lambda\right)_{\Re} \rightarrow\left(E^{*} v, u_{0}\right)_{\Re} \quad \text { as } \quad \lambda \rightarrow 0 .
$$

From (19.15) combined with (20.5) for $w_{\mathrm{\lambda}}=w_{0}$ we then deduce that relation

$$
\left(E^{*} v, u_{0}\right)=\left(v, u_{1}\right)_{\infty}
$$

holds. In view of the definition of the space $\mathcal{G}_{3}$ given in Section 9 , the latter statement is equivalent to saying that the function $u_{0}$ lies in $\mathcal{G}_{3}$ and satisfies $E u_{0}=f_{0}, T u_{0}=0$. Theorem $9_{3}^{I}$, which states that the modified weak and the strong extension of the operator $E$ coincide, now implies that the function $u_{0}$ lies in $\mathfrak{F}(A D)$. We may formulate the result as

LEMMA $20_{3}$ : Let the matrices $B_{\lambda}^{r}$ approach mod $\mathrm{J}$ the matrices $B^{r}$ for which $\sum_{\rho} B^{\rho}=B$; let $f_{\lambda}$ be functions in $\mathfrak{\subseteq}_{\mathcal{A}}$ with $S M h_{\lambda}=0$, and let $\left\|f_{\lambda}\right\|_{A}$ be bounded. Further let $u_{\lambda}$ be the solution of the difference equation $E_{\lambda} u_{\lambda}=f_{\lambda}$ and satisfy the initial condition (10.4). Then a function $u_{0}$ in $\mathfrak{F}(A D)$ with $T u_{0}=0$ exists such that for an appropriate sequence $\lambda \rightarrow 0$ relations

$$
\begin{gathered}
\left(w, u_{\lambda}\right)_{\mathscr{Q}} \rightarrow\left(w, u_{0}\right)_{\Re}, \\
\left(w, E E_{\lambda} u_{\lambda}\right)_{\mathscr{Q}} \rightarrow\left(w, E u_{0}\right)_{\mathfrak{Q}}
\end{gathered}
$$

hold with every function $w$ in $\mathfrak{S}$.

\section{Differentiated Difference Operator}

The preceding result will be applied to two particular operators, in place of $E_{\lambda}$, namely to $\Delta_{l} E_{\lambda}$ and to $\Delta_{l}$.

In doing this we specify $B_{\lambda}^{r}$ by

$$
B_{\lambda}^{r}=B^{r}=m^{-1} B,
$$

$B$ being assumed to have bounded continuous derivatives in $R$. We assume that the function $h$ is in $\mathfrak{F}(D)$ and vanishes on $\mathfrak{s}$, i.e. $S h=0$; furthermore we set

$$
f_{\lambda}=f \text {. }
$$


We then apply the operation $\Delta_{l}$ to equation (19.13) and obtain

$$
A^{\rho} \Delta_{\rho} \Delta_{l} u_{\lambda}+\Delta_{l} A^{\rho} \cdot[l] \Delta_{\rho} u_{\lambda}+[l] B^{\rho} \cdot[\rho] \Delta_{i} u_{\lambda}+\Delta_{l} B^{\rho} \cdot[\rho] u_{\lambda}=\Delta_{l} f .
$$

The coefficients occurring in this expression are defined at points $y$ in $R$ for which $[l] y$ is in $Q$. If $[l] y$ is not in $\Omega$ we define $[l] B^{r}=B^{r}, \Delta_{l} B^{r}=0, \Delta_{l} A^{r}=0$. If $y$ but not $[r] y$ is in $R$, also $[l][r] y$ is not in $a$, as seen from the remark made in Section 19. Therefore, we have

$$
[r] \Delta_{l} u(y)=0 \quad \text { when }[r] y \text { is outside } \Re \text {. }
$$

Equation (21.2) can be considered an equation of the type treated in Section 19. To this end we consider systems of functions $\hat{u}=\left[u, u_{\rho}\right]$ with the inner product $(\hat{v}, \hat{u})=(v, u)+\left(v_{\mu}, u_{\mu}\right)$. To such systems we assign the matrices

$$
\hat{A}^{r}=\left(\begin{array}{cccc}
A^{r} & 0 & . & 0 \\
0 & A^{r} & \cdot & 0 \\
\cdot & \cdot & \cdot & \cdot \\
0 & 0 & \cdot & A^{r}
\end{array}\right\} \text {, }
$$

as in (18.2); but instead of $\hat{B}$ we introduce the matrices

$(21.4) \quad \hat{B}_{\lambda}^{r}=\left(\begin{array}{cccc}\Delta_{1} B^{r} & \delta_{1}^{r} \Delta_{1} A^{!}+[1] B^{r} & \cdot & \delta_{1}^{r} \Delta_{1} A^{m} \\ \cdot & \cdot & \cdot & \cdot \\ \Delta_{m} B^{r} & \delta_{m}^{r} \Delta_{m} A^{1} & \cdot & \delta_{m}^{r} \Delta_{m} A^{m}+[m] B^{r}\end{array}\right) \cdot$

Here $\delta_{m}^{r}$ is the Kronecker symbol.

Since the matrices $A$ and $B$ have bounded derivatives a constant $\hat{b}$ exists such that

$$
\left|\hat{B}_{\lambda}^{r}\right| \leq \hat{b}, \quad r=1, \cdots, m \text {; }
$$

cf. (19.10). The constants $a$ and $c$, cf. (2.2) and (19.21), may serve as constants $\hat{a}$ and $\hat{c}$.

When we set

$$
\begin{gathered}
\hat{u}_{\lambda}=\left[u_{\lambda}, \Delta_{l} u_{\lambda}\right], \\
\hat{f}_{\lambda}=\left[f, \Delta_{l} f\right],
\end{gathered}
$$

cf. (21.1), equations (21.2) and (19.13) can be combined to

$$
\hat{E}_{\lambda} \hat{u}_{\lambda}=\hat{f}_{\lambda} \text {. }
$$


Relations (21.3) and (19.4) take the place of (19.4). Inequality (19.27) therefore holds in the form

$$
\left\|\hat{u}_{\lambda}\right\|_{a} \leq \gamma\left\|\hat{f}_{\lambda}\right\|_{a}
$$

or, by (21.6), (21.7),

$$
\left(u_{\lambda}, u_{\lambda}\right)_{\mathscr{R}}+\left(\Delta_{\rho} u_{\lambda}, \Delta_{\rho} u_{\lambda}\right)_{\mathscr{R}} \leq \gamma^{2}(f, f)_{\mathscr{R}}+\gamma^{2}\left(\Delta_{\rho} f, \Delta_{\rho} f\right)_{\mathscr{R}} .
$$

The results of Section 20 are also applicable. For, the matrix $\hat{B}_{\lambda}^{r}$ approaches mod $\mathfrak{I}$ the matrix

$$
\hat{B}^{r}=\left(\begin{array}{cccc}
B^{r} & 0 & \cdot & \cdot \\
D_{1} B^{r} & \delta_{1}^{r} D_{1} A^{!}+B^{r} & \cdot & \delta_{1}^{r} D_{1} A^{m} \\
\cdot & \cdot & \cdot & \cdot \\
D_{m} B^{r} & \delta_{m}^{r} D_{m} A^{!} & \cdot & \delta_{m}^{r} D_{m} A^{m}+B^{r}
\end{array}\right) .
$$

We observe that the requirement $(20.1), \sum^{\rho} \hat{B}^{\circ}=\hat{B}$, is met when $\hat{B}$ is the matrix defined by (18.2). The norms $\|\left.\hat{f}_{\lambda}\right|_{\hat{A}_{\Omega}}$ of the right members $\hat{h}_{\lambda}$ defined by (21.5) are bounded by virtue of (19.8) since $T h=0$.

Lemma $20_{3}$ now shows that a function $\hat{u}_{0}$ in $\hat{\mathfrak{F}}(A D)$ exists with $\hat{T} u_{0}=0$ such that, for a sub-sequence $\lambda \rightarrow 0$,

$$
\begin{aligned}
\left(\hat{w}, \hat{u}_{\lambda}\right)_{\Re} & \rightarrow\left(\hat{w}, \hat{u}_{0}\right)_{\Re} \\
\left(\hat{w}, \hat{E}_{\lambda} \hat{u}_{\lambda}\right)_{\Re} & \rightarrow\left(\hat{w}, \hat{E} \hat{u}_{0}\right)_{\Re},
\end{aligned}
$$

$\hat{w}$ being any function in $\mathfrak{S}$. Relation (21.10) in particular, implies

$$
\begin{aligned}
\left(w, u_{\lambda}\right)_{\Re} & \rightarrow\left(w, u_{0}\right)_{\mathscr{R}}, \\
\left(w, \Delta_{z} u_{\lambda}\right)_{\mathscr{R}} & \rightarrow\left(w, u_{02}\right)_{\mathscr{R}},
\end{aligned}
$$

$w$ being any function in $\mathfrak{S}$.

On the other hand, we may apply Lemma $20_{3}$ to the operator $D_{l}$, instead of $E$, with $f_{\lambda}=\Delta_{l} u_{\lambda}$ since $\left\|\Delta_{l} u_{\lambda}\right\|_{\Omega}$ is bounded by virtue of (21.10). Hence a function $u_{l 0}$ in $\mathfrak{F}\left(D_{l}\right)$ exists with $S u_{l 0}=0$ such that

$$
\begin{aligned}
& \left(w, u_{\lambda}\right)_{R} \rightarrow\left(w, u_{l 0}\right)_{\mathscr{R}}, \\
\left(w, \Delta_{l} u_{\lambda}\right)_{\mathscr{R}} & \rightarrow\left(w, D_{l} u_{30}\right)_{\mathbb{R}} \quad \text { as } \quad \lambda \rightarrow 0 .
\end{aligned}
$$

Comparison of (21.16) vith (21.14) yields $u_{t 0}=u_{0}$, comparison of (21.17) with (21.15) yields $u_{0 l}=D_{l} u_{l 0}=D_{l} u_{0}$. Thus it is proved that $u_{0}$ is in $\mathfrak{F}\left(D_{l}\right)$, hence in $\mathfrak{F}(D)$, and that

$$
\hat{u}_{0}=\hat{D} u_{0} .
$$

Further, we note that $\hat{D} u_{0}$ is in $\mathfrak{F}(A D)$ with $S \hat{D} u_{0}=0$. 
Relation (21.13) implies, in particular, the relation $\left(w, E_{\lambda} u_{\lambda}\right)_{\mathscr{R}} \rightarrow\left(w, E u_{0}\right)_{\mathfrak{R}_{\mathrm{K}}}$ as $\lambda \rightarrow 0$, whence, because of $E_{\lambda} u_{\lambda}=f$, the identity $(w, f)_{\mathscr{R}}=\left(w, E u_{0}\right)_{\mathscr{R}}$ results.

Since $w$ is arbitrary, this means $E u_{0}=f$. Using the uniqueness of the solution $u$ with $T u=0$ of equation $E u=f$, with which Theorem $17_{s}^{0}$ is concerned, we obtain the identification

$$
u_{0}=u \text {. }
$$

Thus it follows that the function $u$ lies in $\mathfrak{F}(A D D)$.

From the definitions (21.7) of $\hat{f}_{\lambda}$ and (18.4) of $\hat{D} f$, combined with relation (19.9), which holds because of $S f=0$, we deduce the relation

$$
\left\|\hat{f}_{\lambda}-\hat{D} f\right\|_{\Re} \rightarrow 0 \quad \text { as } \quad \lambda \rightarrow 0 .
$$

Now, $\hat{f}_{\lambda}=\hat{E}_{\lambda} \hat{u}_{\lambda}$ by (21.8). Hence relation (21.13) yields

$$
(\hat{w}, \hat{D} f)_{\mathscr{R}}=\left(\hat{w}, \hat{E} \hat{u}_{0}\right)_{\mathfrak{R}} \text {. }
$$

Since $\hat{w}$ is arbitrary in $\hat{\mathfrak{G}}$, we conclude

$$
\hat{E} \hat{D} u=\hat{E} \hat{u}_{0}=D f=\hat{D} E u .
$$

Since this relation implies relation (18.9) and hence (17.4), Theorem $17_{\mathrm{s}}^{0}$ is proved.

\section{Reduction of the General to the Special Differentiability Theorem}

It is likely that the general Differentiability Theorem 17 can be proved, just as the special one, by employing solutions of finite difference equations. A difficulty would arise only in prescribing values of the solution at points of the form $[r] y$ and $[r][l] y$, so as to simulate prescribing on the surface $s$ initial data together with their derivatives. This difficulty can be avoided by reducing the general to the special differentiability theorem.

Naturally, one will try such a reduction by subtracting from the function $u$ a function $i$ so chosen that the special differentiability theorem is applicable

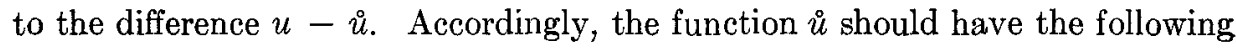
properties:

A) $\stackrel{i}{u}$ agrees with $u$ on $\mathrm{s}$,

$$
S i=S u=S g
$$

B) $\stackrel{u}{u}$ is in $\mathfrak{F}(A D)$.

C) $E$ i agrees with $E u=f$ on $s$,

$$
S E i=S E u=S f
$$

D) Eri is in $\mathfrak{F}(D)$.

It is easy to construct such a function $i$ if the matrices $A$ and $B$ and the functions $g$ and $f$ possess higher differentiability properties than have been 
required so far. We first assume these properties and then eliminate them by an approximation procedure.

These properties are

1) The matrices $A^{r}(y)$ and $B(y)$ belong to $\mathfrak{C}_{2}$,

2) The function $g(y)$ belongs to $\mathbb{E}_{3}$,

3) The function $f(y)$ belongs to $\mathbb{E}_{2}$.

If these conditions are satisfied we simply set

$$
\dot{u}=g-\sigma\left[A^{\rho} \sigma_{\rho}\right]^{-1}[E g-f] .
$$

Here $\sigma_{r}(y)$ are the quantities introduced in Section 17 in connection with condition IV. Clearly, this function $\check{\varkappa}(y)$ satisfies conditions $A, B$, and, since

$$
E \mathfrak{u}=f-\sigma E\left[A^{\rho} \sigma_{\rho}\right]^{-1}[E g-f],
$$

also conditions $C$ and $D$.

The special Theorem $17_{\S}^{0}$ is therefore applicable to the function $u-\dot{u}$. It yields that this function, and hence the function $u$ itself, belongs to $\mathfrak{F}(D)$. It yields further that the relations

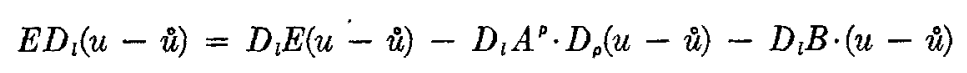

and

$$
S D_{l}(u-\ddot{u})=0
$$

hold. By virtue of $S E u=S E i$, the latter relation is equivalent with

$$
S D_{l}(u-\ddot{u})=S\left(A^{\rho} \sigma_{\rho}\right)^{-1} \sigma_{l}(E u-E \ddot{u}) .
$$

We know that relation (17.4) holds for the function $\dot{u}$, since this relation is a formal identity for all functions which possess second derivatives $D_{l} D_{\tau} u=$ $D_{r} D_{l} u$. From the remarks made in connection with relation (17.6) we infer that also relation (17.5) is a formal identity for all functions $g$ and $u$ in $\mathfrak{F}(D)$ with $S u=S g$; therefore this relation holds for $u$ in place of $u$. Hence we may conclude from (22.5), (22.6) that the function $u$, with which Theorem $17_{\mathrm{s}}$ is concerned, satisfies relations (17.4) and (17.5). Thus Theorem $17_{\mathrm{s}}$ is reduced to Theorem $17_{\mathrm{s}}^{0}$, provided conditions 1 to 3 are satisfied.

Before we can eliminate these conditions we must establish an a priori inequality valid for the solution $u$ which exists under these conditions.

To this end we use the notions introduced in Section 18 and write equations (17.4), (17.5) in the form

$$
\begin{gathered}
\hat{E} \hat{D} u=\hat{D} f, \\
S \hat{u}=S\left\{\hat{D} g-\left(A^{p} \sigma_{p}\right)^{-1} \sigma(f-E g)\right\},
\end{gathered}
$$

cf. (18.9), (18.10). Then we use inequality (7.1) $s$ for $\hat{E}$ instead of $E$ and find

$$
\|\hat{D} u\|_{\alpha}^{2} \leq \gamma^{2}\|\hat{D} f\|_{Q}^{2}+\gamma^{2}\left\|\hat{D} g-\left(A^{\rho} \sigma_{\rho}\right)^{-1} \sigma(f-E g)\right\|_{\delta}^{2} .
$$


Note that the constant $\gamma$ depends only on the bounds $|A|_{\mathcal{A}},\left|\left(\sum_{\rho} A^{\rho}\right)^{-1}\right|_{\text {A }}$, $\left|D_{r} A^{r}\right|_{\Omega},|B|_{G}$ and on the domain $R$; obviously the same constant $\gamma$ can be taken if $R$ is replaced by $R_{\tau}$ for any $\tau>0$. Using relations (17.6) we may derive the inequality

$$
\|\hat{D} u\|_{\Re}^{2} \leq \eta\|\hat{D} f\|_{\Re}^{2}+\eta\|f\|_{s}^{2}+\eta\|\hat{D} g\|_{\S}^{2}
$$

in which $\eta$ is a constant which depends on bounds for $\left|A^{r}\right|,\left|\left(A^{\rho} \sigma_{\rho}\right)^{-1}\right|,\left|\sigma_{r}\right|$, and $|B|$ on $s$.

We let $\kappa$ be a positive number such that

$$
A^{\rho} \sigma_{\rho} \geq \kappa^{-1} \text { on } S \text {. }
$$

We then select a number $\tau>0$ and consider the domain $\Omega_{r}$ introduced in Section 1. We approximate the functions $A^{r}(y), B(y)$ uniformly in $\mathbb{R}_{\tau}$ by functions $A_{\nu}^{\tau}(y), B_{v}(y)$ satisfying the conditions formulated in Section 2 which are in $\mathfrak{S}_{2}$ and for which

$$
A^{\rho} \sigma_{\rho} \geq(2 \kappa)^{-1} \text { on } S .
$$

Next we approximate the functions $f$ and $g$ by functions $f_{v}$ in $\mathfrak{F}\left(D^{2}\right)$ and $g_{v}$ in $\mathfrak{F}\left(D^{3}\right)$ such that

$$
\begin{aligned}
& \left\|\hat{D} f_{\nu}-\hat{D} f\right\|_{A_{r}}+\left\|f_{v}-f\right\|_{s_{r}} \rightarrow 0, \\
& \left\|\hat{D} g_{\nu}-\hat{D} g\right\|_{s_{r}} \rightarrow 0 \quad \text { as } \quad \nu \rightarrow \infty .
\end{aligned}
$$

According to the Existence Theorem $6_{s}^{E}$ a solution $u_{\nu}$ of the equations $E_{v} u_{v}=f_{\nu}$, $S u_{v}=S g_{v}$ exists. The statement of Theorem $17_{\delta}$ holds for this solution $u_{\nu}$ and inequality (22.7) is valid in the form

$$
\left\|\hat{D} u_{\nu}\right\|_{\hat{H}_{\tau}}^{2} \leq \eta\left\|\hat{D} f_{\nu}\right\|_{R_{\tau}}^{2}+\eta\left\|f_{\nu}\right\|_{\delta_{\tau}}^{2}+\eta\left\|\hat{D} g_{v}\right\|_{\delta_{r}}^{2} \text {. }
$$

The constant $\eta$ actually depends on the subscript $\nu$ and on the domain $\mathbb{R}_{r}$; but evidently, we may replace it by a common constant, independent of $\nu$ and $\tau$.

From the sequence $\hat{u}_{v}=\hat{D} u_{v}$ we now may select a subsequence which converges weakly to a limit function $\hat{u}_{0}$ defined in $\mathfrak{R}_{r}$,

$$
\left(\hat{w}, \hat{D} u_{\nu}\right)_{\mathscr{G}_{r}} \rightarrow\left(\hat{w}, \hat{u}_{0}\right)_{\mathbb{G}_{r}} \quad \text { as } \quad \nu \rightarrow \infty .
$$

Clearly, the function $\hat{u}_{0}$ can be defined with reference to the whole domain $R$ so as to satisfy the inequality

$$
\left\|\hat{u}_{0}\right\|_{\Theta} \leq \eta\left\|\hat{D}_{f}\right\|_{\Theta}+\eta\|f\|_{s}+\eta\|\hat{D} g\|_{s} .
$$

Since also $S u_{v}=S g=S u$ it is clear that the function $u_{0}$ lies in $\mathfrak{C}(D)$ and that.

$$
\hat{D} u_{0}=\hat{u} \text {. }
$$

From the identity $\mathfrak{C}_{\S}=\mathfrak{F}$ it follows that $u_{0}$ lies in $\mathfrak{F}(D)$. 
We next use relation

$$
\left(\hat{E}_{\nu}^{*} \hat{w}, \hat{u}_{\nu}\right)_{R_{r}}=\left(\hat{w}, \hat{D} f_{\nu}\right)_{Q_{r}}-\left(\hat{w}, \hat{\sigma} E_{\nu} g\right)_{s_{\tau}}+\left(\hat{w}, \hat{\sigma} f_{\nu}\right)_{s_{\tau}}+(w, \hat{D} g)_{\delta_{r}},
$$

which holds for any function $\hat{w}$ in $\hat{\mathfrak{E}}_{1}$ which vanishes on $\mathfrak{J}_{\tau}$. Since $\left\|\hat{u}_{\nu}\right\|_{\alpha_{\tau}}$ is bounded we may conclude from it and (22.8) that relation

$$
\left(\hat{E}^{*} \hat{w}, \hat{u}_{0}\right)_{\mathbb{R}_{\tau}}=(\hat{w}, \hat{D} f)_{\mathbb{R}_{\tau}}+(w, \hat{D} g)_{s_{\tau}}-(\hat{w}, \hat{\sigma} E g)_{s_{\tau}}+(\hat{w}, \hat{\sigma}, f)_{s_{\tau}}
$$

holds. Since functions $w$ in $\hat{\mathfrak{C}}_{1}$ with $T \hat{w}=0$ can uniformly be approximated by functions in $\mathbb{S}_{1}$ which vanish on $J_{r}$ it follows that the latter relation holds for $\hat{w}$ in $\mathfrak{G}_{1}$ with $T \hat{w}=0$. Hence $\hat{u}_{0}$ lies in $\hat{\mathfrak{S}}_{\mathfrak{s}}(A D)$ and satisfies the relations $\hat{E} \hat{u}_{0}=$ $\hat{D} f, S \hat{u}_{0}=S(D g-\sigma E g+\sigma f)$. From the uniqueness of the solution of $E u=f$, $S u=S_{g}$ it is clear that $u_{0}$ is identical with $u$. Thus Theorem $17_{\mathrm{g}}$ is proved.

It may be mentioned that Theorem $17 \mathrm{~s}$ could have been proved in a different way, by reducing it to Theorem $17{ }_{3}^{0}$ for the adjoint $\hat{E}^{*}$ of the operator $\hat{E}$, cf. (18.3). However, the procedure employed here seems to be simpler.

\section{BiBLIOGRAPHY}

[1] Weber, H., Die partiellen Differentialgleichungen der mathematischen Physik nach Riemann's Vorlesungen in 4ter Auflage neu bearbeitet, Braunschweig, Friedrich Vieweg, 1900 , Volume 1, p. 390.

[2] Hadamard, J., Sur l'intégrale résiduelle, Bull. Soc. Math. France, Vol. 28, 1900, pp. 69-90.

[3] Zaremba, S., Sopra un teorema d'unicità relativo alla equazione delle onde sferiche, Rend. Accad. Naz. Lincei, Ser. 5, Vol. 24, 1915, pp. 904-908.

[4] Rubinowicz, A., (A) Herstellung von Lösungen gemischter Randwertprobleme bei hyperbolischen Differentialgleichungen zweiter Ordnung durch Zusammenstückelung aus Lösungen einfacherer gemischter Randwertaufgaben, Monatsh. Math. Phys., Vol. 30, 1920, p. 65, (B) Über die Eindeutigkeit der Lösung der Maxwellschen Gleichungen, Phys. Zeit., Vol. 27, 1926, p. 707, (C) Zur Integration der Wellengleichung auf Riemannschen Flächen, Math. Ann., Vol. 96, 1927, pp. 648-687.

[5] Friedrichs, K., and Lewy, H., (A) Über die Eindeutigkeit und das Abhängigkeitsgebiet der Lösungen beim Anfangswertproblem linearer hyperbolischer Differentialgleichungen, Math. Ann., Vol. 98, 1927, pp. 192-204, (B) Über fortsetzbare Anfangsbedingungen bei hyperbolischen Differentialgleichungen in drei Veränderlichen, Nachr. Ges. Wiss. Göttingen, No. 26, 1932, pp. 135-143. Courant, R., Friedrichs, K., and Lewy, H., (C) Über die partiellen Differenzengleichungen der mathematischen Physik, Math. Ann., Vol. 100, 1928, pp. 32-74.

[6] Schauder, J., Das Anfangswertproblem einer quasilinearen hyperbolischen Differentialgleichung zweiter Ordnung in beliebiger Anzahl von unabhängigen Veränderlichen, Fund. Math., Vol. 24, 1935, pp. 213-246.

[7] Sobolev, S. L., (A) Diffractsia vol i na rimanovykh poverkhnostakh, Akad. Nauk, SSSR, Trudy Mat. Inst. V. A. Steklova, Vol. 9, 1935, pp. 39-105, (B) Methode nouvelle à résoudre le problème de Cauchy pour les équations linéaires hyperboliques normales, Rec. Math. (Mat. Sbornik), N. S. 1(43), 1936, pp. 39-72.

[8] Frankl, F., Über das Anfangswertproblem für lineare und nichtlineare hyperbolische partielle Differentialgleichungen zweiter Ordnung, Rec. Mathématique (Mat. Sbornik), N. S. 2(44), 1937, pp. 793-814.

[9] Petrovskii, I., Uூber das Cauchysche Problem für Systeme von partiellen Differentialgleichungen, Rec. Math. (Mat. Sbornik) N. S. 2(44), 1937, pp. 814-868. 
[10] Christianovitch, S., Le problème de Cauchy pour les equations non lineaires hyperbaliques, Rec. Math. (Mat. Sbornik), N. S. 2(44), 1937, pp. 871-897.

[11] Sobolev, S. L., Sur la théorie des équations hyperboliques aux dérivées partielles, Rec. Math. (Mat. Sbornik), N. S. 5(47), 1939, pp. 71-99.

[12] Friedrichs, K. O., (A) On differential operators in Hilbert space, Amer. J. Math., Vol. 61, 1939, pp. 523-544, (B) The identity of weak and strong extensions of differential operators, Trans. Amer. Math. Soc., Vol. 55, 1944, pp. 132-151.

[13] Leray, J., (A) Lectures on hyperbolic equations with variable coeffcients, Princeton, Institute for Advanced Studies, Fall, 1952, (B) On linear hyperbolic differential equations with variable coefficients on vector spaces, Princeton, Annals of Mathematics Studies, Volume 33 , in preparation.

[14] Holmgren, E., Über Systeme von linearen partiellen Differentialgleichungen, Öfvers. Kongl. Vetens.-Akad. Förh., Vol. 58, 1901, pp. 91-103.

[15] Hadamard, J., Lectures on Cauchy's Problem in linear partial differential equations, Yale University Press, 1923, or Le problème de Cauchy et les équations aux dérivées partielles linéaires hyperboliques, Hermann, Paris, 1932.

[16] Riesz, M., L'intégrale de Riemann-Liouville et le problème de Cauchy, Acta Math., Vol. 81, 1948, pp. 1-222.

[17] Bureau, F., Quelques questions de Géometrie suggérées par la theorie des équations aux dérivées partielles totalement hyperboliques, Colloque de Géométrie Algébrique, Liége, 1949.

[18] Fourès-Bruhat, Y., Theorème d'existence pour certains systémes d'équations aux dérivées partielles non linéaires, Acta Math., Vol. 88, 1952, pp. 141-225.

[19] Lax, P. D., The initial value problem for non-linear hyperbolic equations in two independent variables, Contribution to the Theory of Partial Differential Equations, Princeton University Press, Annals of Mathematics Studies, No. 33, in preparation. 